\title{
Observations and scaling of travelling bubble cavitation
}

\author{
By Y. KUHN DE CHIZELLE 1 , S. L. CECCIO \\ AND C. E. BRENNEN ${ }^{1}$ \\ ${ }^{1}$ Division of Engineering and Applied Science, California Institute of Technology, Pasadena, \\ CA 91125, USA \\ ${ }^{2}$ Department of Mechanical Engineering and Applied Mechanics, University of Michigan, \\ Ann Arbor, MI 48109, USA
}

(Received 3 February 1994 and in revised form 13 October 1994)

Recent observations of growing and collapsing bubbles in flows over axisymmetric headforms have revealed the complexity of the 'micro-fluid-mechanics' associated with these bubbles (van der Meulen \& van Renesse 1989; Briançon-Marjollet et al. 1990; Ceccio \& Brennen 1991). Among the complex features observed were the bubble-tobubble and bubble-to-boundary-layer interactions which leads to the shearing of the underside of the bubble and alters the collapsing process. All of these previous tests, though, were performed on small headform sizes. The focus of this research is to analyse the scaling effects of these phenomena due to variations in model size, Reynolds number and cavitation number. For this purpose, cavitating flows over Schiebe headforms of different sizes $(5.08,25.4$ and $50.8 \mathrm{~cm}$ in diameter) were studied in the David Taylor Large Cavitation Channel (LCC). The bubble dynamics captured using high-speed film and electrode sensors are presented along with the noise signals generated during the collapse of the cavities.

In the light of the complexity of the dynamics of the travelling bubbles and the important bubble/bubble interactions, it is clear that the spherical Rayleigh-Plesset analysis cannot reproduce many of the phenomena observed. For this purpose an unsteady numerical code was developed which uses travelling sources to model the interactions between the bubble (or bubbles) and the pressure gradients in the irrotational flow outside the boundary layer on the headform. The paper compares the results of this numerical code with the present experimental results and demonstrates good qualitative agreement between the two.

\section{Introduction}

The focus of this paper is on travelling bubble cavitation and the interactions between the flow and the bubbles which occur in this type of cavitating flow. It is motivated by the fact that these interactions radically alter the dynamics of bubble collapse and therefore the noise and damage potential of that process.

The dynamics of collapsing cavitation bubbles have received much attention since it was first recognized that the violence of the collapse was responsible for cavitation damage. The mechanisms of shock wave production during rebound (Gilmore 1952; Hickling \& Plesset 1964) and re-entrant microjet shocks (Naude \& Ellis 1961; Benjamin \& Ellis 1966; Lauterborn \& Bolle 1975; Fujikawa \& Akamatsu 1980; Shima et al. 1981, 1983; Kimoto 1987) have been extensively explored both experimentally and analytically. However, virtually all of these observations and analyses have 
focused on bubble collapse in a quiescent liquid despite the fact that a number of experimenters have commented on the deformation of cavitation bubbles by the flow (see, for example, Knapp \& Hollander 1948; Parkin 1952; Ellis 1952; Blake, Wolpert \& Geib 1977). Until the recent work of van der Meulen \& van Renesse (1989), Briançon-Marjollet, Franc \& Michel (1990), Ceccio \& Brennen (1991) and Kumar \& Brennen (1993) the nature and consequences of this deformation had not been examined. It is now clear that the deformation and fission caused by the interaction of the bubble with the nearby solid surface and with the pressure gradients and shear in the flow play a very important role in the dynamics and acoustics of travelling bubble cavitation. This paper presents the results of a series of experiments design to explore the scaling of these phenomena as well as the results of a computational effort to model some of the observed interactions.

It is important to establish the relationship between the size of a typical cavitation bubble and the thickness of the laminar boundary layer near the minimum pressure point. For a body with a typical dimension, $l$, in a flow of typical velocity, $U$, the ratio of the thickness of the laminar boundary layer, $\delta$, to the typical maximum radius of a travelling cavitation bubble, $R_{M}$, will be given by

$$
\frac{\delta}{R_{M}}=\frac{R e^{-1 / 2}}{2\left(-\sigma-C_{p m i n}\right)},
$$

where the cavitation number, $\sigma=2\left(p_{\infty}-p_{v}\right) / \rho U^{2}$, the minimum coefficient of pressure, $C_{p \min }=2\left(p_{\text {min }}-p_{\infty}\right) / \rho U^{2}, p_{\infty}, p_{v}$ and $p_{\text {min }}$ are respectively the free-stream pressure, the vapour pressure and the minimum pressure in the flow in the absence of cavitation and $R e$ denotes the Reynolds numbers, $R e=U l / \nu$. Therefore, provided $\left(-\sigma-C_{p m i n}\right)$ is of the order of 0.1 or greater, it follows that for the high Reynolds numbers which are typical of most of the flows in which cavitation is a problem, the boundary layer is usually much thinner than the typical dimension of the bubble.

The experiments of Ceccio \& Brennen (1991) were performed on $5.08 \mathrm{~cm}$ diameter axisymmetric head-forms and revealed a surprising complexity in the flow around a single cavitation bubble. It was observed that the bubbles have a spherical-cap shape and are separated from the solid surface by a thin film of liquid. This general conformation persists during the growth phase, though with the larger bubbles the thin film appears to become unstable and may begin to shear off the underside of the bubble leaving a cloud of smaller bubbles behind in the wake. The collapse phase is quite complex and consists of at least three processes occurring simultaneously, namely collapse, shearing due to the velocity gradient near the surface and the rolling up of the bubbles into vortices as a natural consequence of the first two processes. These processes tend to produce small transverse vortices with vapour/gas-filled cores. It was noted that the collapse phase was dependent on the shape of the headform and the details differed between the ITTC headform (Lindgren \& Johnsson 1966) which possesses a laminar separation and the Schiebe body (Schiebe 1972; Meyer, Billet \& Holl 1989) which does not. On the ITCC headform, when some of the larger bubbles pass the point of laminar separation they induce 'streaks' of local attached cavitation at the lateral extremes of the bubble. These streaks stretch out as the bubble proceeds downstream, being anchored at the upstream end to a point on the body surface near the laminar separation line. The main bubble then collapses, leaving the two streaks which disappear at a later time.

One of the important consequences of these variations in the details of the collapse processes is the effect on the noise produced by a single cavitation event (Ceccio \& Brennen 1991; Kumar \& Brennen 1993). Bubble fission can produce several bubble 


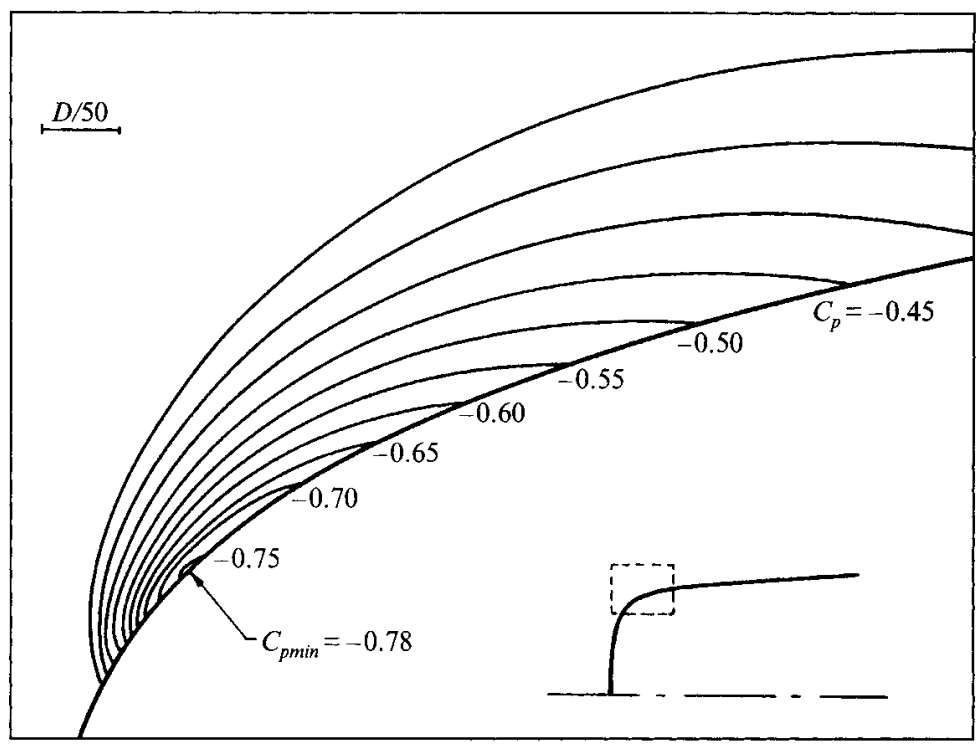

FIGURE 1. Isobars in the vicinity of the minimum pressure point on the axisymmetric Schiebe headform with values of the pressure coefficient, $C_{p}$, as indicated. The pressures were obtained from a potential flow calculation. The insert shows the headform shape and the area that has been enlarged in the main figure (dashed lines).

collapses and therefore several acoustic pulses. Presumably this would also effect the cavitation damage potential of the flow. Kumar \& Brennen (1993) have further examined the statistical properties of the acoustical signals from individual cavitation bubbles on two different headforms in order to learn more about the bubble/flow interactions. All of these previous experiments were, however, conducted in the same facility with the same headform size $(5.08 \mathrm{~cm}$ in diameter) and over a fairly narrow range of flow velocities (around $9 \mathrm{~m} \mathrm{~s}^{-1}$ ). Clearly this raises the issue of how the phenomena identified change with speed, scale and facility. The experiments described here represent one effort to answer some of these questions for the case of cavitation occurring on simple axisymmetric headforms. This paper will focus on travelling bubble cavitation, and the interaction between the flow and the dynamics and acoustics of individual bubbles.

Questions regarding the scaling of cavitation have been raised for many years (see, for example, Holl \& Wislicenus 1961; Billet \& Holl 1979) and even more so in the aftermath of the ITTC comparative tests conducted by Lindgren \& Johnsson (1966) which showed how disparate the appearance of cavitation was at different speeds, in different facilities and at different water 'qualities'. This characterization refers to the number of cavitation nuclei present in the water, where the nuclei usually consist of very small air bubbles in the range of 5 to $300 \mu \mathrm{m}$. As O'Hern, D'Agostino \& Acosta (1988) have shown, the nuclei are similar in size distribution in most deaerated water tunnels and in the ocean. This causes one set of scaling questions since the ratio of nucleus size to the body size will change with the geometric size of the body. The other set of scaling issues derives from the complex interactions between the bubbles and the flow close to the headform, which is Reynolds-number dependent. Scaling effects will thus be caused by the changes in both body size and tunnel velocity. In order to address this problem, a series of experiments were conducted in the Large Cavitation Channel of the David Taylor Research Center in Memphis, Tennessee.

The current investigation utilized an axisymmetric headform shape known as a 
'Schiebe' body (Schiebe 1972) which has been widely used for cavitation research (Meyer et al. 1989; Ceccio \& Brennen 1991). This headform shape has therefore become a useful touchstone with which to compare cavitation data taken from various research laboratories. It exhibits a relatively smooth adverse pressure gradient and the laminar boundary layer therefore does not separate. As part of the computational effort described below, a panel method was developed to solve the axisymmetric potential flow around this headform. This potential flow solution yields the isobars in the low-pressure region on the surface of the headform which are presented in figure 1. The minimum pressure coefficient is $C_{p \min }=-0.78$. Note the large pressure gradient normal to the surface of the headform in the vicinity of the minimum pressure point; we shall see that this significantly distorts the cavitation bubbles. Also note the elongated shape of the low-pressure region near the surface.

\section{Description of the experiments}

The experiments were conducted in a new facility, the Large Cavitation Channel, which has just been constructed for the David Taylor Research Center (Morgan 1990). Briefly this facility is a very large recirculating water tunnel with a working section which is $3.05 \mathrm{~m} \times 3.05 \mathrm{~m}$ in cross-section. It is capable of tunnel speeds above $15 \mathrm{~m} \mathrm{~s}^{-1}$ and the pressure control allows operation at sufficiently low pressures in the working section to permit cavitation investigations. Three Schiebe headforms of diameter, $D$, equal to $5.08,25.4$ and $50.8 \mathrm{~cm}$ were machined out of solid blocks of clear lucite and were mounted in the centre of the working section using after-bodies and a supporting strut as shown in figure 2. The instrumentation used was similar to that of Ceccio \& Brennen (1991) for all three headforms and consisted of a series of surface electrodes and an internally mounted hydrophone. The interiors of the headforms were hollowed out in order to place the hydrophone in the centre and as close as possible to the cavitation. The acoustical path between the cavitation and the hydrophone was made as clean and reverberation-free as possible by fabricating the headforms out of lucite and filling the interior of the headform with water.

In addition, silver epoxy electrodes were flush-mounted on the surface of the headforms as can be seen in the photographs of figures 5 and 6 . A pattern of alternating voltages is applied to the electrode pairs, and the electric current from each electrode is monitored. When a bubble passes over one of the electrodes, the impedance of the flow is altered, causing a drop in current (Ceccio \& Brennen 1991). Thirteen of these electrodes take the form of small circular patches (about $1 \mathrm{~mm}$ in diameter) at different axial locations. The analog signal from the electrode bridge is processed, allowing individual bubbles to be detected. A trigger pulse is then fed to both the digital recorders and to the cameras.

Two cameras, triggered simultaneously, were used to take flash pictures of individual cavitation bubbles at different angles and different enlargements. Four EG\&G model SS166 flash heads with SS162-165 energy storage racks were used. The film exposure time was the flash duration and was measured to be about $30 \mu \mathrm{s}$. Triggering was effected either manually or through a computer-controlled lock-out system connected to the electrode peak detector signal. A variable delay unit was employed in order to take photographs of bubbles at various times after passing an electrode. The EG\&G flash heads were also used in a strobing mode, synchronized with the video camera framing rate (30 f.p.s.) in order to make video recordings for each operating condition.

An International Transducer Corporation hydrophone (model ITC-1042) with a flat isotropic response of $\pm 2 \mathrm{~dB}$ out to $80 \mathrm{kHz}$ was installed on the axis inside each of the 


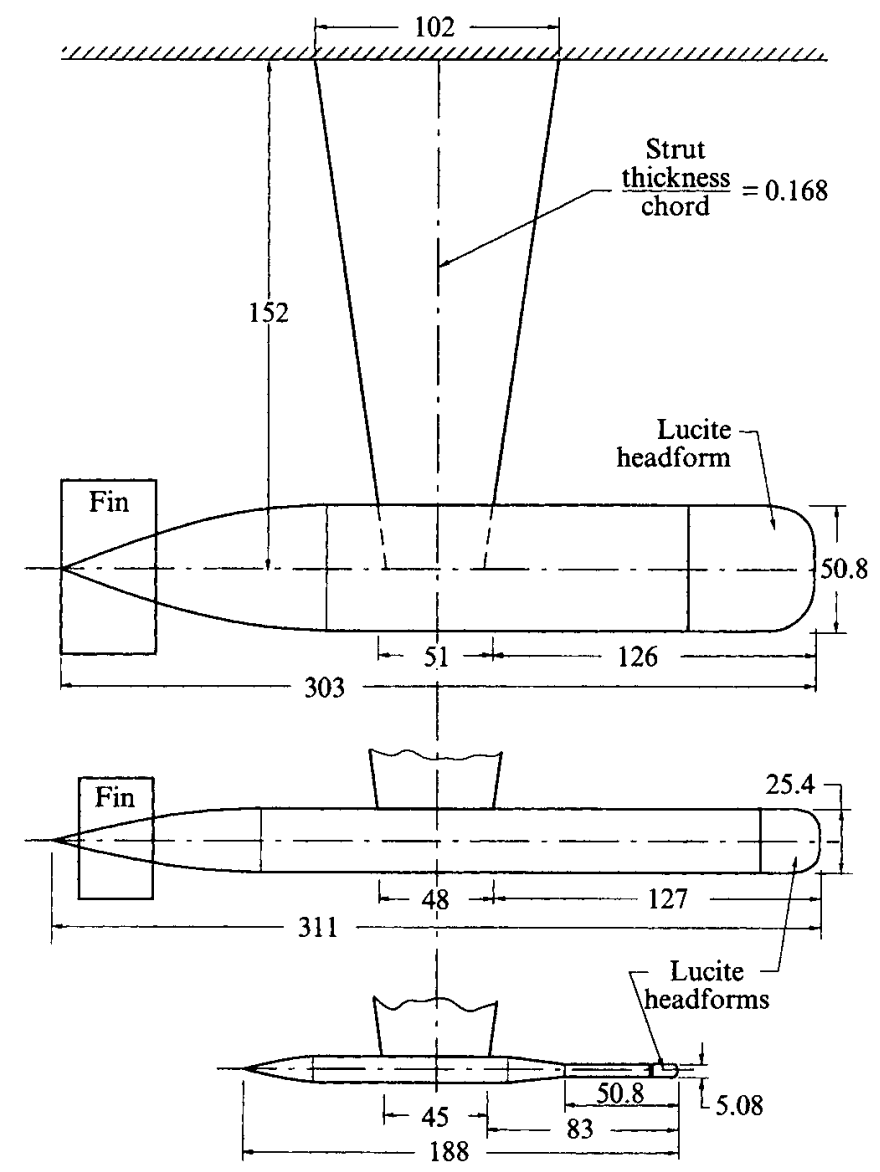

FIGURE 2. Schematic of the system used to support the three headforms in the Large Cavitation Channel. Dimensions in $\mathrm{cm}$.

headforms. The centre of the hydrophone was one headform radius from the front stagnation point. The hydrophone signals were sampled at a rate of $1 \mathrm{MHz}$ with a 12 bit resolution. Ceccio (1990) validated this technique of mounting the hydrophone inside the headform by comparing the signals recorded from a single cavitation event with those recorded by a hydrophone mounted on the tunnel wall. In the present tests, a similar check was performed by comparing the internal hydrophone signals with those from two Sonatech STI-01-02 hydrophones (with a flat frequency response up to $100 \mathrm{kHz}$ ) flush-mounted in recesses in the sidewall of the test section, one upstream and the other downstream of the headform. A spectrum analyser was used to measure the transfer function between these hydrophones for frequencies ranging from 3 to $100 \mathrm{kHz}$. Using the ITC and STI hydrophones in turn as transmitters and receivers, reciprocity calibrations were performed. Furthermore, the specification curves supplied by the hydrophone manufacturers allow us to verify the voltage-to-pressure transfer gain between the hydrophones. Results of these calibration are presented in detail in Kuhn de Chizelle (1993). In summary, the reciprocity calibrations proved accurate to within a $\pm 5 \mathrm{~dB}$ noise level and the voltage-to-pressure transfer gain checked to within $\mathrm{a} \pm 8 \mathrm{~dB}$ noise level.

Two bypass water lines connected to Orbisphere probes monitored the dissolved oxygen content of the tunnel water which was adjusted using the deaeration system of 


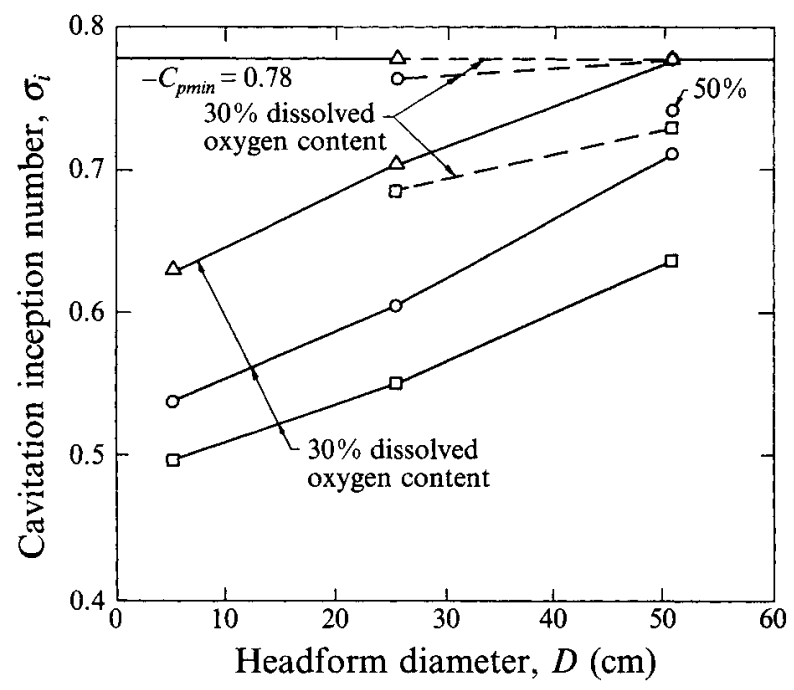

FIGURE 3. Cavitation inception numbers $\sigma_{i}$ for the three different headforms at three tunnel velocities of $9 \mathrm{~m} \mathrm{~s}^{-1}(\triangle), 11.5 \mathrm{~m} \mathrm{~s}^{-1}(O)$, and $15 \mathrm{~m} \mathrm{~s}^{-1}(\square)$, and two dissolved oxygen contents.

the LCC. Values quoted herein refer to the percentage of the saturation value at atmospheric pressure. The water temperature was constantly monitored. Efforts were also made to measure the nuclei content of the tunnel water. A susceptibility meter developed by the David Taylor Research Center (Shen, Gowing \& Pierce 1984) was connected to the tunnel and cavitation susceptibility measurements were made by counting the number of cavitation events per volume of water passing through the venturi, over a range of cavitation numbers and flow rates. Counting was accomplished by the means of a high-frequency hydrophone (band-passed between 10 and $100 \mathrm{kHz}$ ) located next to the venturi of the susceptibility meter.

All three headforms were tested using a similar test matrix which included three dissolved oxygen contents $(80 \%, 50 \%$ and $30 \%$ of saturation), three velocities, $U(9$, 11.5 and $15 \mathrm{~m} \mathrm{~s}^{-1}$ ) and about five different cavitation numbers. Thus the Reynolds number, $R e=U D / \nu$, ranged from $0.54 \times 10^{6}$ to $9.41 \times 10^{6}$.

\section{Cavitation inception}

Figure 3 presents the observed cavitation inception numbers, $\sigma_{i}$, as a function of the headform diameter, $D$, tunnel velocity, $U$, and dissolved oxygen content. Inception was based on an arbitrarily chosen event rate of about 50 cavitation events per second as detected by the first of the flush-mounted patch electrodes. The curves in figure 3 appear to asymptote to a value equal to the magnitude of the minimum pressure coefficient on the surface of the headform $\left(C_{p \min }=-0.78\right)$. As expected, the inception number increases with increasing headform size since the number of nuclei passing through the low-pressure region increases with the larger headforms. Therefore, for a specific event rate, the value of $\sigma_{i}$ is larger. The values of $\sigma_{i}$ also increase with an increase in the dissolved oxygen content for a similar reason.

The differences in the cavitation inception numbers for the three headforms were apparent when photographs at the same cavitation number and flow velocity were compared. At a cavitation number of $\sigma=0.51$, the smallest headform is close to inception and the occasional travelling bubble is observed. In contrast, the $25.4 \mathrm{~cm}$ 
headform displays both travelling bubbles and patch-type cavities at $\sigma=0.51$ and the $50.8 \mathrm{~cm}$ headform exhibits quite extensive cavitation. Thus the scaling effects with headform size were clearly evident.

Figure 3 also shows that the cavitation inception number increases with decreasing tunnel velocity. The reason for this scale effect is less clear. However, in order to achieve the same cavitation number at a lower velocity the tunnel pressure must be reduced. Therefore it may be that the nuclei concentration in the tunnel increases considerably with decreasing operating pressure. In their experiments using a $5.08 \mathrm{~cm}$ headform, Hamilton, Thompson \& Billet (1982) observed the same trend as the velocity was increased (their incipient cavitation numbers of about 1.1 were substantially larger than those presented here).

\section{Cavitation event rates}

Both the photographs and the videotapes were analysed in order to examine the variations in the cavitation event rates with headform size and tunnel velocity. The average number of events was obtained by counting the number of individual bubbles (or events) observed in a single video frame and averaging this number over many frames. It is valuable to process this information in order to compare the cavitation event rate with that which might be expected knowing the population of cavitation nuclei in the oncoming stream. The first step was to convert the data on the average number of events observed in a snapshot in time into data on the cavitation event rates by using bubble lifetimes obtained from the potential flow calculations (discussed later) and the observed locations of bubble appearance and collapse (presented later in figure 10). These event rate data were then converted to free-stream nuclei concentrations using the following model. First it was assumed that all of the nuclei which pass through an annular streamtube bounded by the headform surface and the streamsurface which just touches the $C_{p}=-\sigma$ isobar (see figure 1) cavitate and form observable bubbles. Using the potential flow calculations, the volume flux of liquid in that streamtube for each of the operating conditions could then be calculated. Dividing the event rate data by this flux we obtain an estimate of the number of activated cavitation nuclei per unit liquid volume. These data are presented in figure 4. It should be noted that there are a number of questionable assumptions and approximations implicit in the derivation of these data. For a more detailed analysis the reader is referred to Liu, Kuhn de Chizelle \& Brennen (1993). Nevertheless it is of value to comment briefly on figure 4 .

It can be seen that the data for different cavitation numbers, headform sizes and tunnel velocities appear to correspond to a nuclei concentration of the order of $0.1 \mathrm{~cm}^{-3}$. The most noticeable deviation from this uniform value occurs at the highest speed $\left(U=15 \mathrm{~m} \mathrm{~s}^{-1}\right)$ with the two larger headforms; the reason for this is unclear. The fact that most of the data appear to correspond to the same nuclei concentration is encouraging because it suggests that a more careful analysis which begins with the same nuclei number distribution and follows each nucleus along its streamline (see Meyer et al. 1989; Ceccio \& Brennen 1991; Liu et al. 1993) might allow synthesis of the event rates and the inception numbers.

It would, of course, be more conclusive if we had been able to monitor the actual nuclei population in a proven and reliable way. However, such instrumentation has only recently been developed. In a later paper we shall present simultaneous measurements of the nuclei population and the cavitation event rate, experiments currently being conducted at Caltech. Some efforts were made to monitor the nuclei 


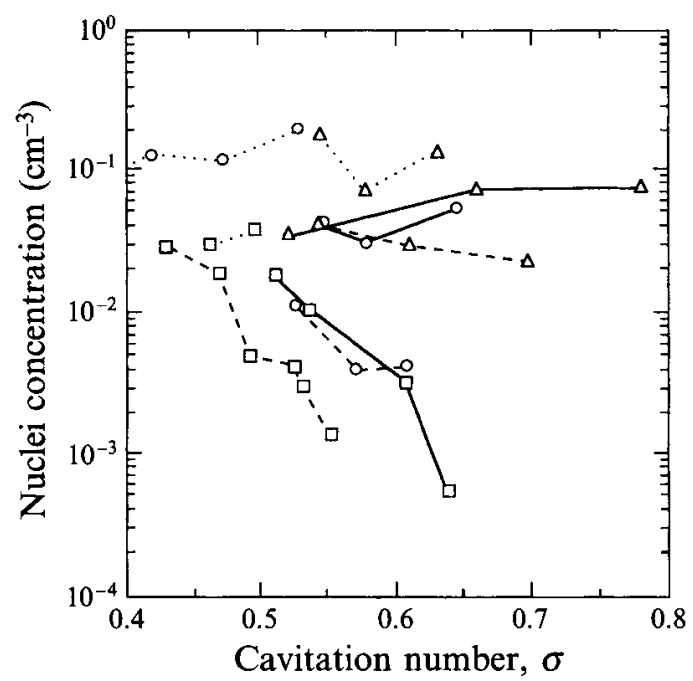

FIGURE 4. Number of excited nuclei per unit liquid volume of the free stream as a function of the cavitation number. Data derived from the observed event rates for the $50.8 \mathrm{~cm}$ headform (solid lines), the $25.4 \mathrm{~cm}$ headform (dashed lines), and the $5.08 \mathrm{~cm}$ headform (dotted lines) at three tunnel velocities of $9 \mathrm{~m} \mathrm{~s}^{-1}(\triangle), 11.5 \mathrm{~m} \mathrm{~s}^{-1}(O)$, and $15 \mathrm{~m} \mathrm{~s}^{-1}(\square)$.

during the LCC experiments by using the aforementioned susceptibility meter which withdrew water from near the bottom of the working section. These qualitative measurements revealed that a sudden increase in the susceptibility occurred when the tunnel pressure was reduced below $60 \mathrm{kPa}$. This correlates with the observation (figure 4) that the event rate increased with the decrease in tunnel pressure needed to achieve the same cavitation number at the lower velocities. More detailed results of these susceptibility measurements can be found in Kuhn de Chizelle (1993).

\section{Cavitation appearance}

A typical cavitation event consists of the growth and collapse of a bubble as it travels through the low-pressure region close to the headform surface. The shape and size which the bubble assumes are dependent on the cavitation number and the local pressure history experienced by the bubble. In this section we shall describe in more detail the evolution of individual bubbles as evidenced by the photographs and video recordings at the same dissolved oxygen content of $30 \%$.

The highest cavitation inception numbers, $\sigma_{i}$, occur for the largest body at the smallest velocities (figure 3 ). Under these conditions the bubble lifetime is short and the bubbles assume a thin disk-like geometry with little or no growth normal to the headform surface as typified by the bubble in figure 5(a). At these cavitation numbers, the critical isobar, $C_{p}=-\sigma$, is very elongated and close to the body surface and the bubble is understandably inhibited from growing beyond this envelope. Collapse appears to proceed by evanescence of the leading edge of the bubble so that the bubble disappears into a certain axial location on the surface.

As the cavitation number is decreased below $\sigma_{i}$, the bubbles grow in volume (in diameter and in thickness) and assume the spherical-cap shape typified by figure $5(b)$. The maximum volume depends primarily on the cavitation number. As the bubbles approach their collapse phase their thickness, $\delta$, normal to the headform surface decreases faster than their base radius, $R$, and the leading edge collapses most rapidly 

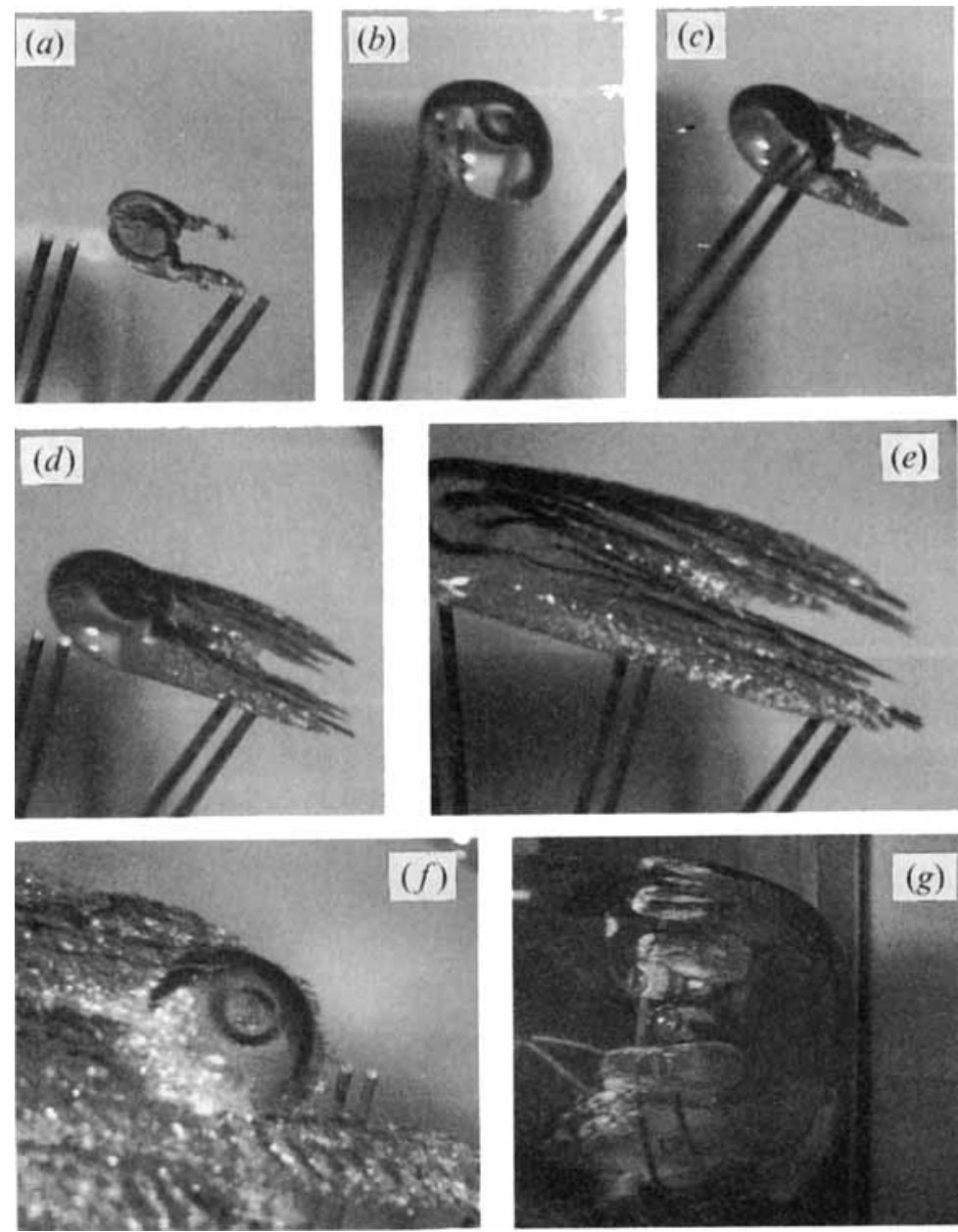

FIGURE 5. Photographs of cavitation events on the $50.8 \mathrm{~cm}$ diameter headform (distance between two patch electrodes is $2.54 \mathrm{~cm}):(a) U=9 \mathrm{~m} \mathrm{~s}^{-1}, \sigma=0.77 ;(b, c, d, e) U=15 \mathrm{~m} \mathrm{~s}^{-1}, \sigma=0.60 ;(f, g)$ $U=15 \mathrm{~m} \mathrm{~s}^{-1}, \sigma=0.54$.

along a fairly straight front (figure $6 a, c$ ). At this stage the bubbles are thin and close to the headform surface as was the case under inception conditions.

One unique feature of the present experiments was the appearance of circular dimples on the exterior surface of the spherical-cap bubbles (figures $5(b, e, f), 6(a, c)$ ). The dimples become more pronounced as the volume of the bubble increases, probably because the relative effects of surface tension are reduced. For this reason they are not readily apparent on the bubbles on the smallest, $5.08 \mathrm{~cm}$, headform or in the experiments of Ceccio \& Brennen (1991). On the largest, $50.8 \mathrm{~cm}$, headform two dimples sometimes occurred on the largest bubbles. The dimples are less noticeable during the growth phase (figure $5 c$ ) and appear early in the collapse phase. Their appearance could be interpreted as a precursor of a classical re-entrant jet but the dimple seems quite stable and remains on the bubble until the last stages of collapse (figure $6 a, c$ ). The reason for the dimple is unclear although the potential flow calculations described below indicate that a local over-pressure forms near the top of the spherical cap early in the growth phase.

Another important phenomenon which occurred was the appearance of the attached 

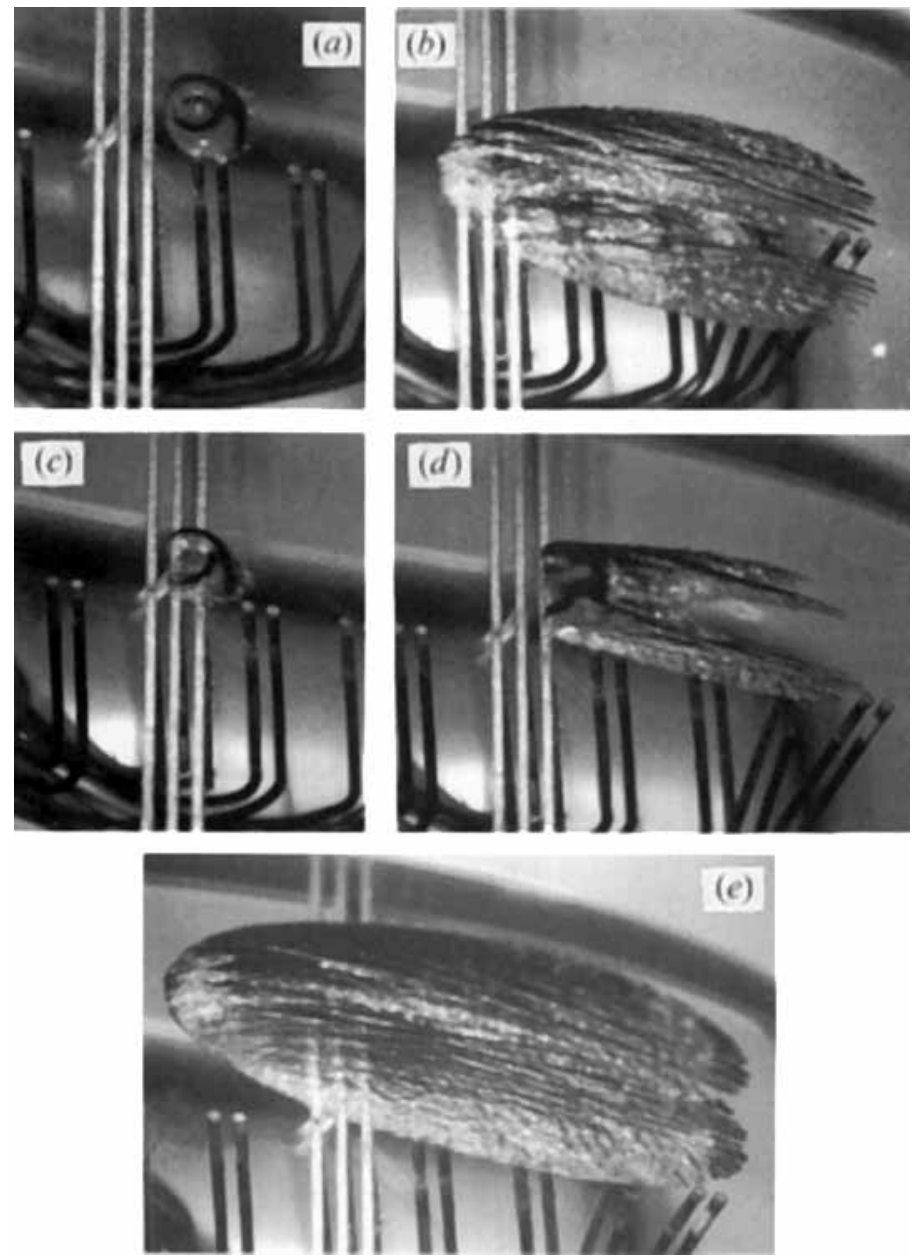

FIGURE 6. Photographs of cavitation events on the $25.4 \mathrm{~cm}$ diameter headform (distance between two patch electrodes is $1.27 \mathrm{~cm}):(a, b) U=15 \mathrm{~m} \mathrm{~s}^{-1}, \sigma=0.55 ;(c, d, e) U=15 \mathrm{~m} \mathrm{~s}^{-1}, \sigma=0.53$.

'streaks' or 'tails' previously described by Ceccio \& Brennen (1991) and exemplified by the bubble shown in figure $5(a)$. The tails are observed to attach at approximately the same axial location on the headform surface (figure $5 c$ ). They extend behind the bubble as it moves downstream and, in the process, increase in both length and thickness (figure $5 c-e$ ). Whether or not a bubble develops tails seems to be determined early in the growth phase. If it does not form tails early in its development (figure $5 c$ ), the bubble will grow and collapse with a smooth, spherical-cap shape (figures $5 b$, $6 a, c)$. When the tails are of small or moderate size, the dynamics of the primary bubble collapse appears unaffected by them as seen in figure $6(d)$. Often, the tails will remain for a brief period after the bubble which created them has collapsed.

The probability that a bubble will develop tails increases as the size of the headform or free-stream velocity is increased. It also increases with decreasing cavitation number, and therefore with increasing size of the bubble. Consequently, the probability appears to correlate with bubble size and/or Reynolds or Weber number. With increasing Reynolds or Weber number, the tails become more extensive, leading to a situation in which the travelling bubble causes cavity separation over its entire "wake' rather than just at the spanwise extremities. Thus, the bubble causes the formation of 
a 'patch' of attached cavitation (figure $6 b, e$ ) similar to the structures previously described by Holl \& Carroll (1979), Huang (1979) and others. Many of these cavitation patches are initiated by the passage of a travelling bubble. Whether this is the case for all of the patches could not be determined from the present study, but that possibility exists. Usually, the patches persist for only a short length of time. However, at the higher Reynolds (or Weber) numbers and lowest cavitation numbers they seemed to persist for a substantial length of time and, in the most extreme cases, remain almost indefinitely. As the cavitation number is reduced over the larger headform, the increasing number of patches merge to create larger attached structures. These are fairly stable and remain on the headform, thus creating attached cavities for periods of up to a few seconds. We note that the transient cavitation patch phenomenon was never observed on the smallest headform. Indeed, that headform exhibits an abrupt switch from travelling bubble cavitation (some of which have trailing tails) to persistent attached cavities.

At cavitation numbers close to the $-C_{p \min }$ no patches and very few bubble tails are observed. Under these conditions the tails seem unable to grow sufficiently to form a patch-like cavity. At lower cavitation numbers the patches become longer and thicker. Notice that the patch in figure $6(b)$ at a larger cavitation number is thinner and does not extend as far downstream as that of figure 6(e). The collapse mechanism of the patches is unclear. In the video recordings they vanish entirely in the interval $(1 / 30 \mathrm{~s})$ between two frames and it was not possible to determine whether the entire patch is swept downstream or whether it collapses down onto the headform.

Finally it is important to emphasize that the trends described above are changes in the probabilities of the occurrence of the different types of events. Thus the video recordings clearly show a wide range of different events occurring at the same operating condition. Even under the conditions at which many patch cavities were observed, some very smooth spherical-cap travelling bubbles still occurred (figure $5 b$, $g$ ). Figures $5(f-h)$ show spherical-cap bubbles riding above or through fully attached cavities. Comparing the shape of the bubbles encountering patch cavities with those which do not, it is clear that the shapes differ because the former are not subjected to the boundary layer shear which the latter experience. Bubbles which do encounter patches or attached cavities will eventually collapse and merge with the attached cavity upstream of its closure region. By doing so they appear to perturb the attached cavity shape, as has been observed by Briançon-Marjollet et al. (1990).

\section{Bubble size and shape}

One of the earliest models of the growth and collapse of cavitation bubbles was proposed by Plesset (1949) who adapted the equations of motion for spherical bubbles originally obtained by Rayleigh (1917) to the problem of travelling bubble cavitation. The nuclei from which the bubble grows is assumed to be a micron-size gas bubble which is initially in equilibrium with the surrounding liquid. Rayleigh-Plesset models have been widely used to predict travelling bubble cavitation (for example, Parkin 1952; Holl \& Kornhauser 1970; Johnson \& Hsieh 1966; Hamilton et al. 1982). In the present study, the pressure/time history, $C_{p}(t)$, on a streamline close to the surface of the Schiebe headform was calculated using the potential flow solution used for figure 1. Assuming this is the pressure at infinity experienced by a nucleus as it is carried along this streamline, the Rayleigh-Plesset equation was numerically integrated to obtain the radius/time history of the cavitation bubble, $R(t)$, for various cavitation numbers and initial nuclei. Typical results for the dimensionless radius of the bubble, $r=R / D$, as a 


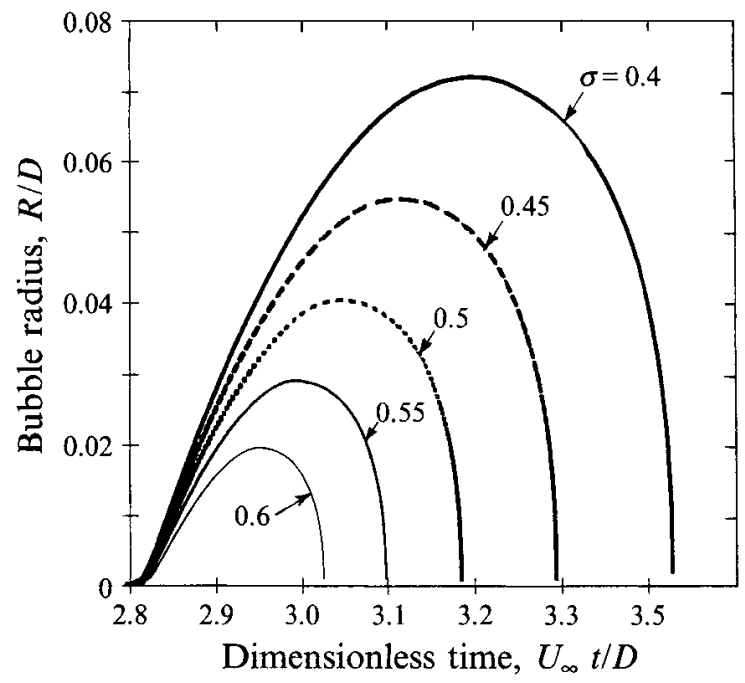

FIGURE 7. Bubble radius as a function of time from the Rayleigh-Plesset equation for various cavitation numbers: $\sigma=0.60,0.55,0.50,0.45$ and 0.40 . The calculations use $W e=93 \times 10^{4}$ and $R e=6.8 \times 10^{6}$. The origin of time is arbitrary

function of the dimensionless time, $\tau=U t / D$, are presented in figure 7 for various cavitation numbers; the following additional parameters were used: initial nucleus size, $R_{0}=100 \mu \mathrm{m}, D=50.8 \mathrm{~cm}$, and $U=11.5 \mathrm{~m} \mathrm{~s}^{-1}$. Note that all of the cavitation numbers used in the calculations of figure 7 resulted in the $100 \mu \mathrm{m}$ nucleus experiencing supercritical tension and therefore undergoing explosive cavitation growth. If the cavitation number were greater than the inception value or if the nucleus were small enough so that it did not experience supercritical conditions at these cavitation numbers then the growth rate would be very much smaller and the nucleus would not result in a macroscopic bubble.

Experimental data on the sizes of the actual cavitation bubbles were obtained from the still photographs and the video tape recordings. The base of an individual bubble (the surface next to the headform) at the point of maximum bubble growth is close to circular and was characterized by its radius $R_{\max }$. We present in figure 8 the ratio of maximum base radius to headform diameter, $R_{\max } / D$ as a function of the cavitation number. We can see that the velocity, $U$, the headform diameter, $D$, have little influence on the non-dimensional bubble size, for a fixed cavitation number. This result can be explained by analysis of the Rayleigh-Plesset equation for spherical bubble growth. Once the nucleus has begun to grow, viscous (Reynolds number) and surface tension (Weber number) effects soon become negligible and the dimensionless bubble growth rate, $(\mathrm{d} R / \mathrm{d} t) / U$ depends only on the cavitation number, $\sigma$, and the pressure coefficient history, $C_{p}(t)$. Moreover, the time available for growth in the low-pressure region scales like $D / U$ and so the equations yield values for $R_{\max } / D$ which depend only on the headform shape (as manifest in $C_{p}$ ) and $\sigma$. Calculations of the Rayleigh-Plesset equation are also included in figure 8 . It is remarkable that, despite the very nonspherical shape of the actual cavitation bubbles, the Rayleigh-Plesset equation yields values which are close to the base radius of the actual bubbles. It is as if the headform surface acts as a plane of symmetry for the growth of the bubble and the pressure distribution parallel to the surface is the sole driving term in that plane. Note, however, that at low cavitation numbers the Rayleigh-Plesset model departs from the experimental data and yields larger bubble sizes. 


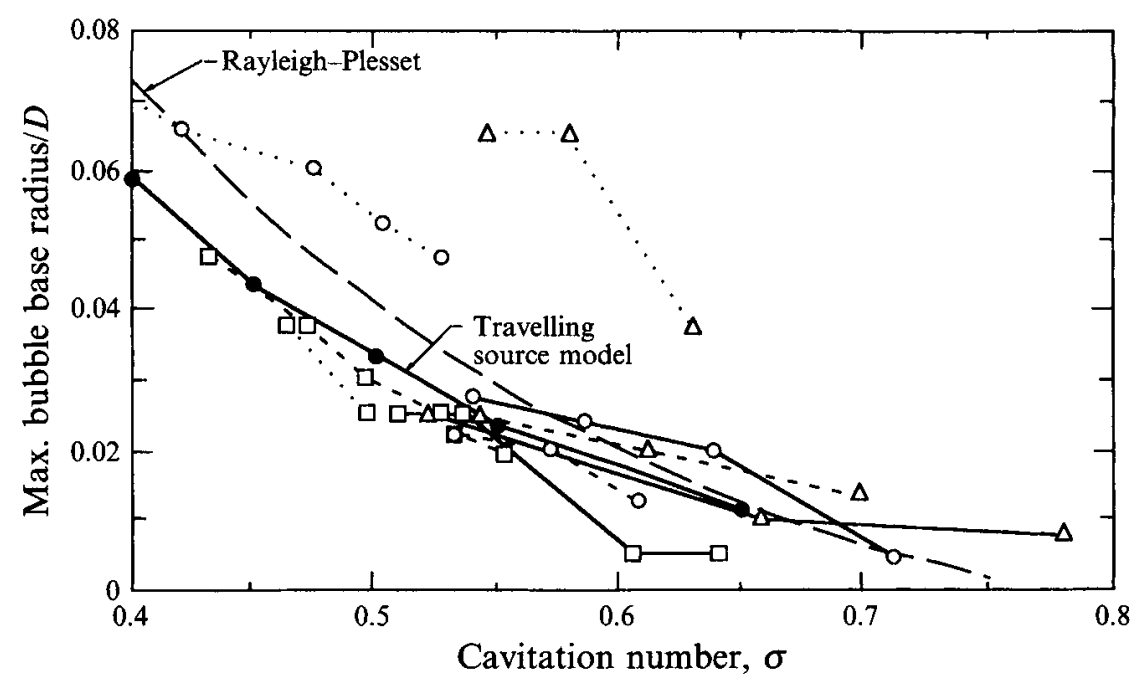

FIGURE 8. The maximum radius of the base of the cavitation bubbles (normalized by $D$ ) as a function of the cavitation number, $\sigma$, for the three headforms: $50.8 \mathrm{~cm}$ (solid line). $25.4 \mathrm{~cm}$ (dashed line), $5.08 \mathrm{~cm}$ (dotted line) at three different tunnel velocities of $9 \mathrm{~m} \mathrm{~s}^{-1}(\triangle), 11.5 \mathrm{~m} \mathrm{~s}^{-1}(O)$, and $15 \mathrm{~m} \mathrm{~s}^{-1}$ ( $\square$ ). Also shown are the results of the Rayleigh-Plesset calculations using the pressure distribution on the surface of the headform (dashed line) and the results of the present travelling source model (solid line with O).

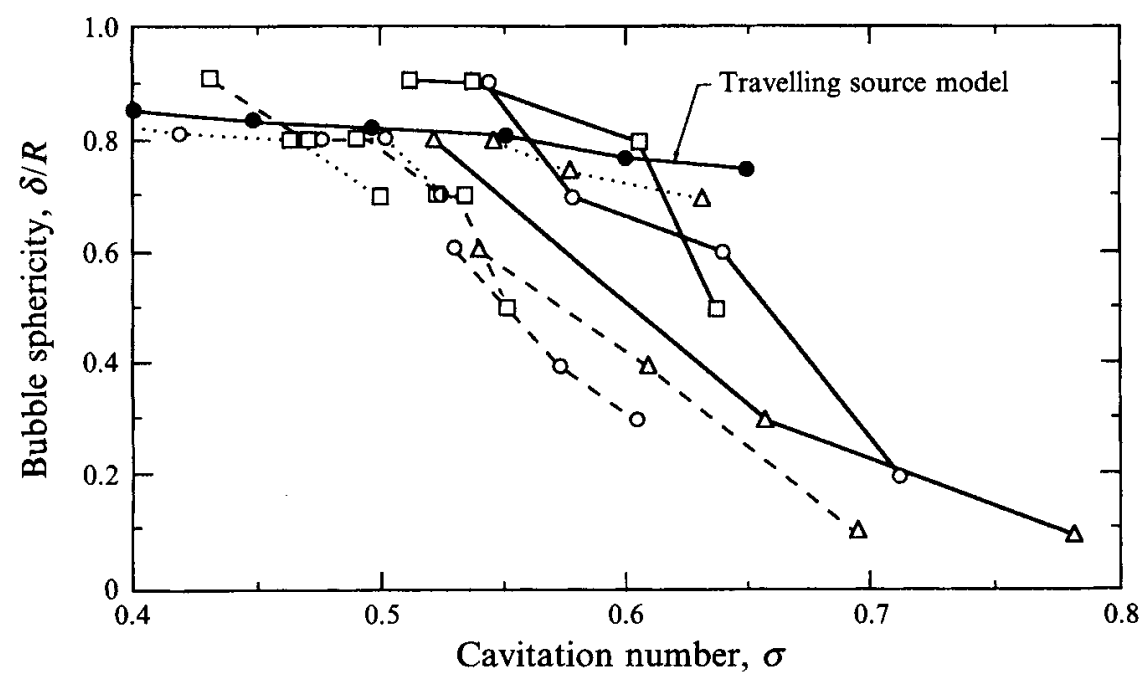

FIGURE 9. The sphericity the cavitation bubbles, $\delta / R$, as a function of the cavitation number, $\sigma$, for the headform diameters and tunnel velocities of figure 8 . Also shown are the results of the present travelling source model.

The only experimental data in figure 8 which differ substantially from the rest are those for the $5.08 \mathrm{~cm}$ headform at $U=9 \mathrm{~m} \mathrm{~s}^{-1}$, but these data were subject to large uncertainty since it proved difficult to focus on and then measure such small bubbles in such a large facility.

The bubble sphericity, defined as $\epsilon=\delta / R$ where $\delta$ is the previously defined bubble thickness and $R$ is the base radius, is presented in figure 9 ; this was evaluated at the point of maximum size of the bubble. On the larger diameter headforms, the bubbles 


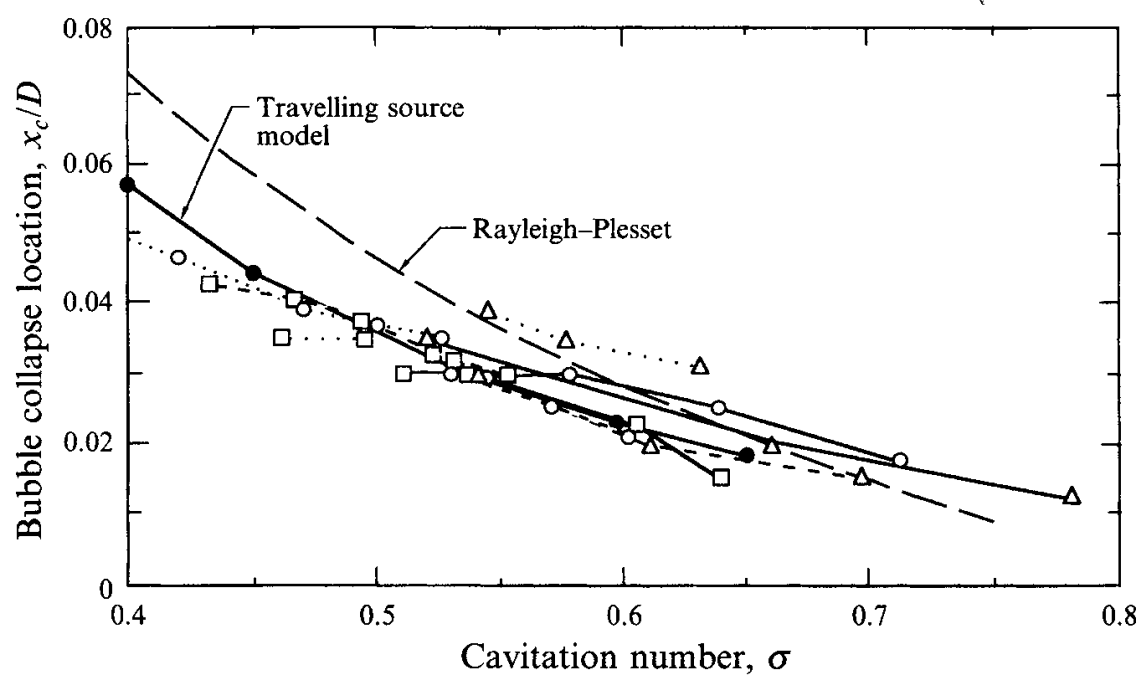

FIGURE 10. The location of the collapse of the cavitation bubbles, $x_{c} / D$, as a function of the cavitation number, $\sigma$, for the headform diameters and tunnel velocities of figure 8. As shown are the results of Rayleigh-Plesset calculations and the present travelling source model.

appeared very thin for cavitation numbers close to inception. Figure 1 demonstrated that the isobars near the minimum pressure point are very elongated and close to the surface. Hence there exists a high-pressure gradient normal to the headform surface. The Rayleigh-Plesset calculations for a nucleus experiencing pressures along a streamline close to the headform show that, for cavitation numbers higher than 0.6 , a bubble would grow to a radius that exceeds the height of the critical isobar $C_{p}=-\sigma$. This over-pressure on the exterior of the bubble forces it to be flattened. For smaller cavitation numbers, the critical isobar is considerably further from the headform surface, allowing it more latitude to grow in the direction normal to the headform surface. The $5.08 \mathrm{~cm}$ headform has a significantly smaller inception number of about 0.55 . All the bubbles observed on this headform are quite hemispherical, presumably because the $C_{p}=-\sigma$ isobar is far from the surface at the low cavitation numbers for which travelling cavitation bubbles could be observed.

Also, since the pressure gradient normal to the surface is much larger than that parallel to the surface, the bubble thickness, $\delta$, decreases faster than the base radius, $R$, during the collapse phase. Therefore, the sphericity of a bubble always decreased during the collapse phase.

As a final comment on the sphericity, we might expect that the bubbles at higher Weber numbers would be more distorted than those at lower values of this parameter. This could be a factor which contributes to the increased probability of attached tails at the higher Weber numbers.

Measurements of the location of bubble collapse are presented non-dimensionally in figure 10 and exhibit a clear dependence on cavitation number with little dependence on the body diameter or the free-stream velocity. Results of the Rayleigh-Plesset calculations are also shown and are in fair agreement with the observations at high cavitation numbers. We note that as soon as the attached cavities appear at low cavitation numbers, the pressure distribution is modified and the bubbles tend to merge into these cavities, making an estimation of the collapse location more difficult.

As the cavitation number is reduced, the number of cavitating bubbles appearing on the surface in a still photograph increases. The fraction of the surface in the low- 


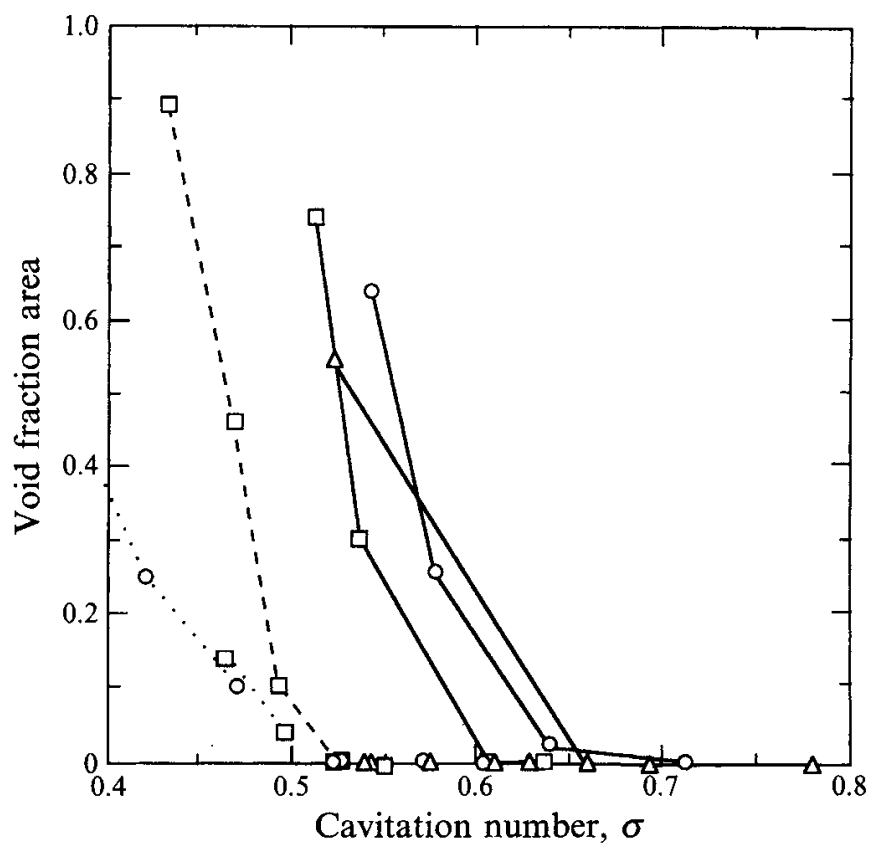

FIGURE 11. Average void fraction area on the surface of the headform as a function of the cavitation number, for velocities and headform diameters as given in figure 8 .

pressure region which is covered by bubbles was estimated from the pictures and plotted against $\sigma$ in figure 11. Note that the increase in the void fraction at lower cavitation numbers is due to the presence of patches and attached cavities. Bubbles do not contribute significantly to this void fraction. The reason for the increased number of patches (or the extent of attached cavitation) for the larger headforms and lower velocities is not clear.

\section{Comparisons with potential flow calculations}

As a preliminary step toward understanding the interactions between a cavitation bubble and the flow field, a computational algorithm was developed (Kuhn de Chizelle \& Brennen 1993) which could be used to investigate the interaction between a bubble and the potential flow around the Schiebe headform. For convenience, we refer to this as the 'travelling source' model. The objective was to focus on the interaction of the bubble with the irrotational flow exterior to the boundary layer and the resulting exterior shape of the bubble. Different, viscous flow analyses would be needed to study the phenomena of the liquid layer instability and triggering of attached cavitation.

The simplest version of the travelling source model is based on the assumption that the perturbations in the irrotational flow caused by the bubble can be approximately modelled by a travelling source of adjustable intensity and position and that, once an image source is added to substantially satisfy the boundary condition on the headform surface, the remaining corrections which are required involve small modifications of the basic structure of the flow. It is, of course, possible to solve the inviscid, irrotational problem by using a boundary integral method in which the surface of the headform and the surface of the bubble are divided into boundary elements. Indeed, Chahine, Duraiswami \& Rebut (1992) made some preliminary calculations of this kind. We 


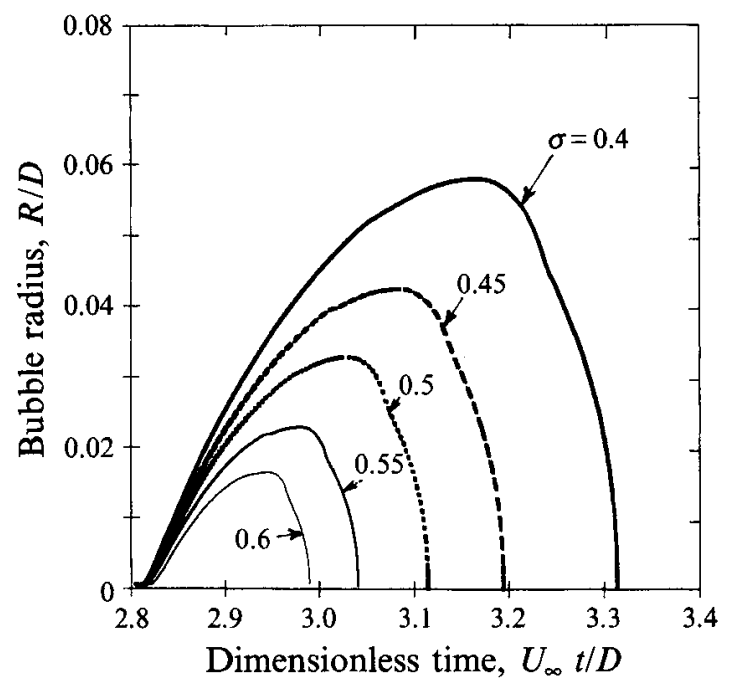

FIGURE 12. Bubble base radius as a function of time from the three-parameter travelling source model for cavitation numbers as follows: $\sigma=0.60,0.55,0.50,0.45$ and 0.40 . The calculations use $W e=93 \times 10^{4}$ and $R e=6.8 \times 10^{6}$. The origin of time is arbitrary.

believe that the travelling source model has the advantage of improved resolution of the bubble dynamics at much reduced computational time. It is also readily extended to allow studies involving more than one bubble so that interaction effects might be examined.

In brief, the travelling source model utilizes a boundary element method to construct the basic axisymmetric flow around the headform; a series of axisymmetric ring panels are deployed for this purpose. In the simplest version, a travelling bubble is simulated by a single point source. The reflected image of this source in the headform surface is added in order to help satisfy the boundary condition on the headform surface. The surface of the bubble is a material surface within the flow and its evolution is tracked using 'marker nodes'. The fluid velocity at each of these marker nodes is calculated from the potential flow solution and the profile at the next time step is obtained using displacements which are the fluid velocities multiplied by the time increment. The rest of the three-dimensional geometry of the bubble can be tracked in the same way. It follows that the kinematic condition on the bubble surface is quite accurately satisfied.

The dynamic condition on the bubble surface is satisfied approximately by choosing the strength and location of the source at each time step in order to satisfy the pressure condition (vapour pressure plus minor adjustments due to surface tension and gas content effects) at the marker nodes as closely as possible. In the simplest version of the method, this involves just three adjustable parameters, namely the strength and coordinate locations of the source. But further sources or singularities can clearly be added to improve the degree to which the dynamic condition is satisfied at the marker nodes. Note that the basic three-parameter version of the algorithm can only be expected to model the simpler features of the bubble geometry and not the complex features associated with a well-developed re-entrant jet. This would require the addition of several more source parameters. For further details the reader is referred to Kuhn de Chizelle (1993) and Kuhn de Chizelle \& Brennen (1993).

Figure 12 presents results from the three-parameter travelling source model for the bubble base radius as a function of time during growth and collapse at several cavitation numbers. Notice that, in comparison with the Rayleigh-Plesset results of 

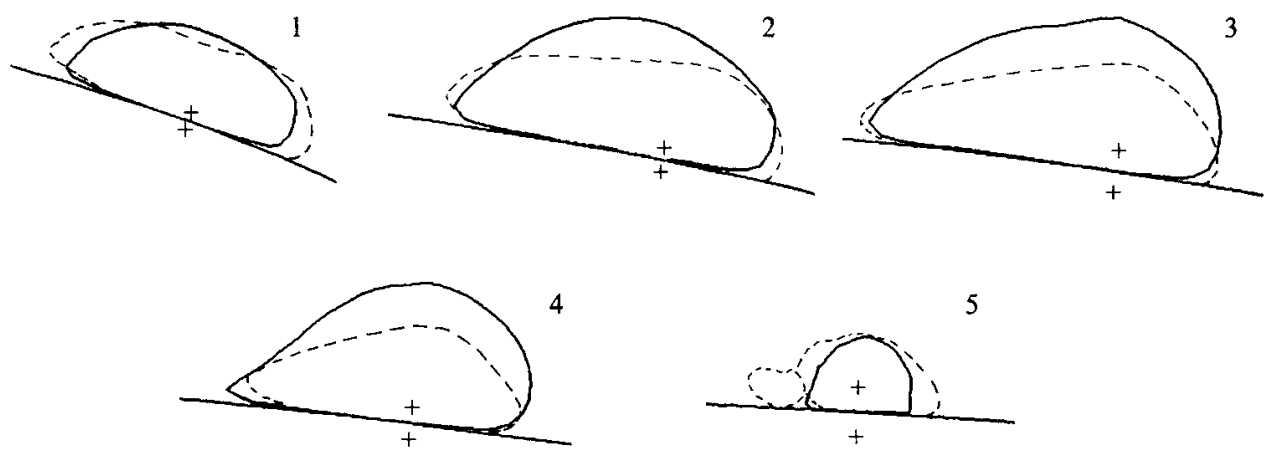

FIGURE 13. Comparison between the profiles of bubbles from the photographs of Ceccio \& Brennen (1991) (dashed lines) and the profiles calculated by the three-parameter version of the travelling source model (solid lines) at five different moments in time during growth and collapse, consecutively numbered 1 to 5 . The flow is from right to left. The locations of the source and the image source are shown by the crosses.

figure 7 , the travelling source model yields smaller bubbles which collapse earlier. The bubbles are smaller because their growth transports their surface to regions of higher pressure further from the headform surface.

In figure 13 the bubble profiles from the photographs of Ceccio \& Brennen (1991) are compared with the profiles computed by the three-parameter version of the travelling source model at the same five moments in time (labelled 1-5) during the bubble evolution. It can be seen that the overall size of the bubbles is in good agreement with the observations and that there is qualitative agreement in the general shape of the bubble as well as the way it changes with time. The program reproduces the spherical-cap shapes which are separated from the headform by a thin liquid layer. During the growth phase we note a minor depression in the top of the cap which is reminiscent of the dimples on top of the bubbles observed by Kuhn de Chizelle et al. (1992b) but not as pronounced. Later the bubble assumes the wedge-like shape similar to the experiments. The computed bubbles are not, however, as elongated as those observed experimentally, particularly at the higher cavitation numbers. The threeparameter version of the travelling source model may not be adequate to simulate such large departures from sphericity.

Values of the maximum base radius computed using the travelling source model are included in figure 8 . The agreement with most of the data is quite satisfactory and shows a significant deviation from the Rayleigh-Plesset calculations described earlier. The sphericity of the bubbles is also shown in figure 9. Here again there is good agreement between the observations and the calculations but only for the lower cavitation numbers where the sphericity is large. As in figure 13, the experimental bubbles at the larger cavitation numbers are significantly flatter.

\section{Bubble cavitation noise}

The radiated acoustic pressure, $p_{a}$, at a large distance, $\mathscr{R}$, from the centre of a bubble of volume $V(t)$ is given by Blake (1986)

$$
p_{a}=\frac{\rho}{4 \pi \mathscr{R}} \frac{\mathrm{d}^{2} V}{\mathrm{~d} t^{2}} .
$$


It is clear that the noise pulse generated at bubble collapse results from the very large and positive values of $\mathrm{d}^{2} V / \mathrm{d} t^{2}$ which occur when the bubble is close to its minimum size. A good measure of the magnitude of the collapse pulse is the acoustic impulse, $I$, defined as the area under the pulse or

$$
I=\int_{t_{1}}^{t_{2}} p_{a} \mathrm{~d} t,
$$

where $t_{1}$ and $t_{2}$ are times before and after the pulse at which $p_{a}$ is zero. It is also useful in the present context to define a dimensionless impulse, $I^{*}$, as

$$
I^{*}=16 \pi I \mathscr{R} / \rho U D^{2} .
$$

We shall compare the experimentally measured values of $I^{*}$ from cavitation bubbles on the headforms of different size with those from numerical calculations of the growth and collapse of bubbles obtained from integration of the Rayleigh-Plesset equation. Details of these calculations are given in Ceccio \& Brennen (1991) and Kuhn de Chizelle et al. $(1992 b)$. For present purposes, we note that variations in the Weber number, Reynolds number and initial size of the nucleus had little effect on the computated impulses (within $\pm 10 \%$ ).

For a range of experimental cavitation numbers, it was possible to identify within the hydrophone output the signal produced by each cavitation event. To isolate individual bubble signals it was found necessary to digitally high-pass filter the signals using a cutoff frequency of $5 \mathrm{kHz}$ in order to reduce the effect of vibration and noise caused by cavitation at the top of the supporting strut. After the processing amplifier transfer function was applied to the filtered signal, the noise from the cavitation was analysed in several ways. Spectral analyses of the cavitation noise were carried out and have been presented in Kuhn de Chizelle et al. (1992a). Here we focus on the analysis of the pressure pulses produced by individual cavitation events. The hydrophone output for each of the experimental conditions was examined in order to identify at least 40 of the larger pulses associated with bubble collapse. The average values of the impulses obtained in this way are plotted against cavitation number in figure 14 (these data are for a $30 \%$ dissolved oxygen content). In viewing these data it must be emphasized that there is considerable variability in the magnitude of the impulses occurring at a particular operating condition. Consequently, the standard deviations corresponding to the averaged $I^{*}$ values of figure 14 are usually between $25 \%$ and $80 \%$ of the average value (for more detail see Kuhn de Chizelle 1993). As in the experiments of Ceccio \& Brennen (1991) the collapse of an individual bubble (or event) seems to be characterized by a fairly well-defined maximum possible value of the impulse but can also produce impulses which are a fraction of this maximum.

Also shown in figure 14 are some data from the $5.08 \mathrm{~cm}$ headform experiments of Ceccio \& Brennen (1991) and a hatched area which encompasses the results from the Rayleigh-Plesset calculations" using the pressure distribution on the surface of the headform. Note first that the upper envelope of the present data for all the headforms and velocities is roughly consistent with the upper envelope for the small-headform data of Ceccio \& Brennen (1991). Perhaps the envelope defined by the present data is somewhat below that of the small-headform data and this may be due to the additional filtering which was necessary in the present experiments. The upper envelopes of both sets of experimental data are approximately one order of magnitude smaller than the impulses obtained from the Rayleigh-Plesset calculations. There are probably two reasons for this. First, the actual maximum volume of the bubbles is significantly 


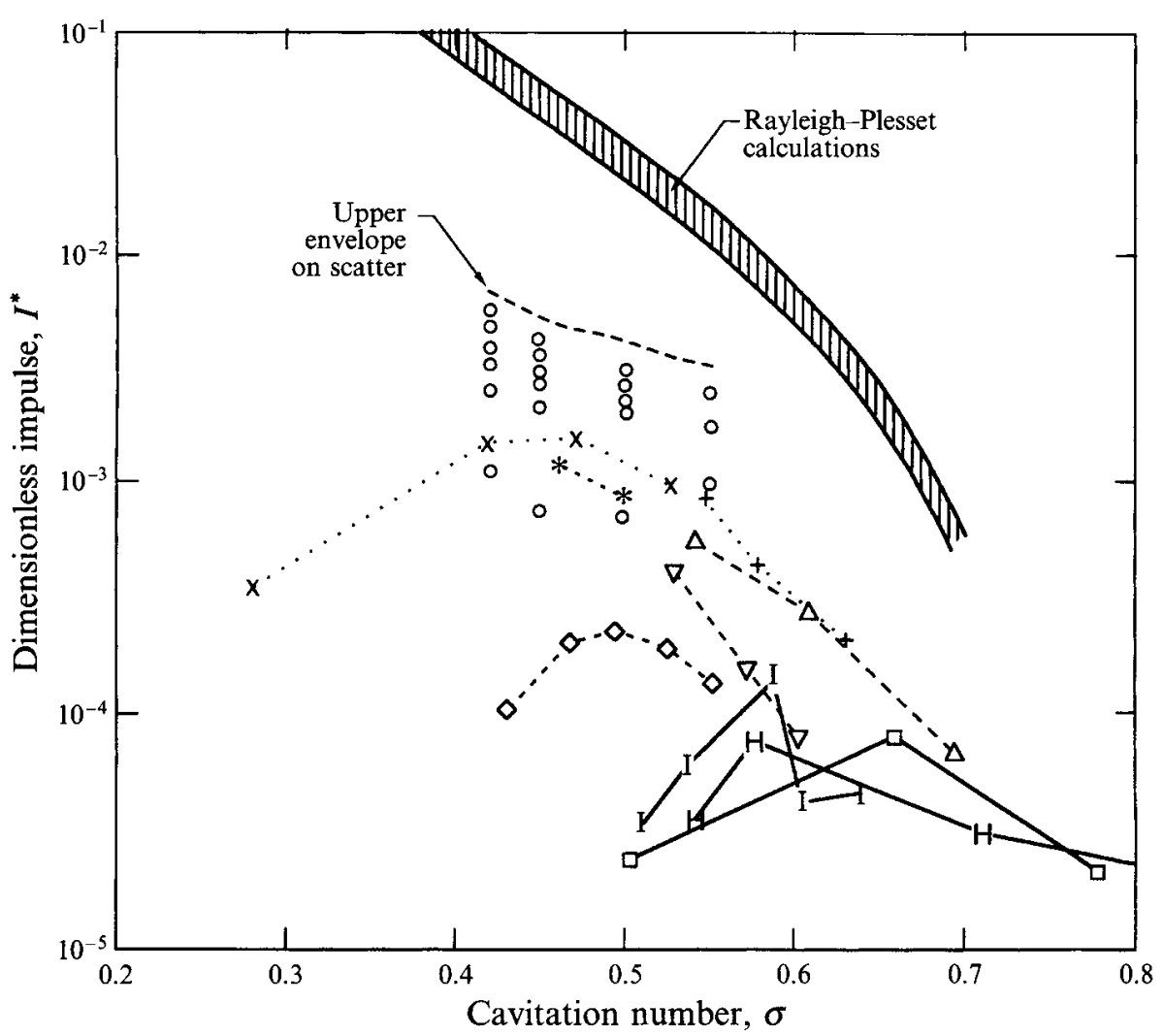

FIGURE 14. The dimensionless impulse, $I^{*}$, as a function of cavitation number, $\sigma$. All the calculations using the Rayleigh-Plesset equation lay within the hatched region. The experimental measurements of Ceccio \& Brennen (1991) on a $5.08 \mathrm{~cm}$ headform (O) are shown along with the upper envelope which was defined by those data. The present results are shown for the three headform diameters $(50.8 \mathrm{~cm}$, thick solid line; $25.4 \mathrm{~cm}$, thin solid line; $5.08 \mathrm{~cm}$, dotted line) at three different tunnel velocities (+, $\triangle, \square, 9 \mathrm{~m} \mathrm{~s}^{-1} ; \times, \nabla, \mathrm{H}, 11.5 \mathrm{~m} \mathrm{~s}^{-1} ; *, \diamond, \mathrm{I}, 15 \mathrm{~m} \mathrm{~s}^{-1}$ ).

smaller than the maximum volume of the Rayleigh-Plesset bubbles as was documented in figures 8 and 9. Since the impulse is correlated with the maximum volume, this is clearly one reason for the discrepancy. This effect can be accounted for, as will shortly be demonstrated. A more speculative, second reason for the discrepancy could be that the more non-spherical the collapse, the less noise might be produced since a spherical collapse may produce the maximum focusing of the unsteady pressures. The interactions of the bubble with the pressure gradients and the boundary layer produce deformations in the shape which, in turn, alter the noise produced.

We anticipated that, perhaps, the acoustic impulses would correlate better with maximum bubble volume than with cavitation number. This correlation has previously been mentioned by many authors including Fitzpatrick \& Strasberg (1956), Hamilton et al. (1982) and Vogel, Lauterborn \& Timm (1989). For this reason the data of figures 8 and 9 were used to calculate the typical maximum bubble volumes for each of the cavitation numbers, tunnel velocities and headform sizes (assuming spherical cap shapes for the bubbles) and the data of figure 14 were then replotted versus maximum cavity volume in figure 15 . Note that the correspondence of the upper envelope of the data is improved over that of figure 14, confirming again that the upper bound on the impulse correlates strongly with the maximum bubble volume. 


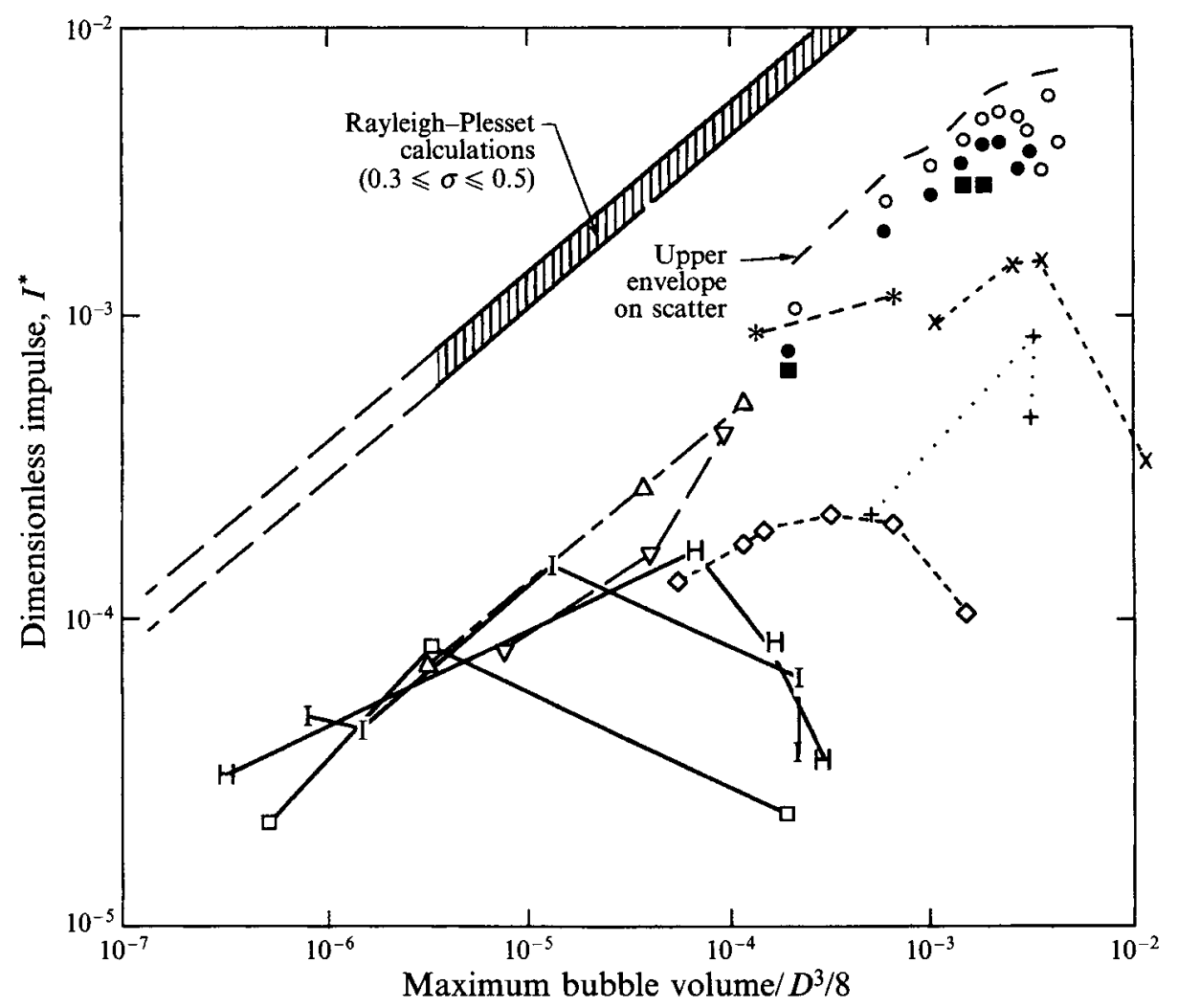

FIGURE 15. The dimensionless impulse, $I^{*}$, as a function of maximum volume of the bubble (divided by $D^{3} / 8$ ). All the calculations using the Rayleigh-Plesset equation for $0.3 \leqslant \sigma \leqslant 0.5$ lay within the hatched region. The experimental measurements on a $5.08 \mathrm{~cm}$ headform by Ceccio \& Brennen (1991) are shown for $\sigma=0.42(O), 0.45(\bigcirc)$ and $0.50(-\square-)$ along with the upper envelope which was defined by those data. The present results are coded as in figure 14 .

The upper envelope on the experimental data in figure 15 and of the Rayleigh-Plesset calculations are both consistent with a relation of the form

$$
I^{*} \approx \beta\left(R_{\max } / D\right)^{5 / 2}
$$

where $\beta$ is some proportionality constant. There is an explanation for the form of this relation which proceeds as follows. From the definitions (2), (3) and (4) one can obtain

$$
I^{*}=\frac{4}{U D^{2}}\left[\left(\frac{\mathrm{d} V}{\mathrm{~d} t}\right)_{t_{2}}-\left(\frac{\mathrm{d} V}{\mathrm{~d} t}\right)_{t_{1}}\right]
$$

If the typical bubble radius at the times $t_{1}$ and $t_{2}$ is denoted by $R_{X}$ and the typical pressure coefficient is denoted by $C_{p x}$ then it follows from the Rayleigh-Plesset equation (by setting $\mathrm{d}^{2} V / \mathrm{d} t^{2}=0$ and evaluating $\mathrm{d} V / \mathrm{d} t$ ) that

$$
I^{*} \approx 32 \pi\left(R_{X} / D\right)^{2}\left(C_{p x}-\sigma\right)^{1 / 2} .
$$

Numerical calculations of the Rayleigh-Plesset equation for a wide range of flows and conditions showed that $R_{X} / R_{\max } \approx 0.62$. Moreover, calculations with the Schiebeheadform pressure distribution showed that $\left(C_{p x}-\sigma\right) \propto R_{\max } / D$. Substituting these two expressions into (7) yields the relation (5). 


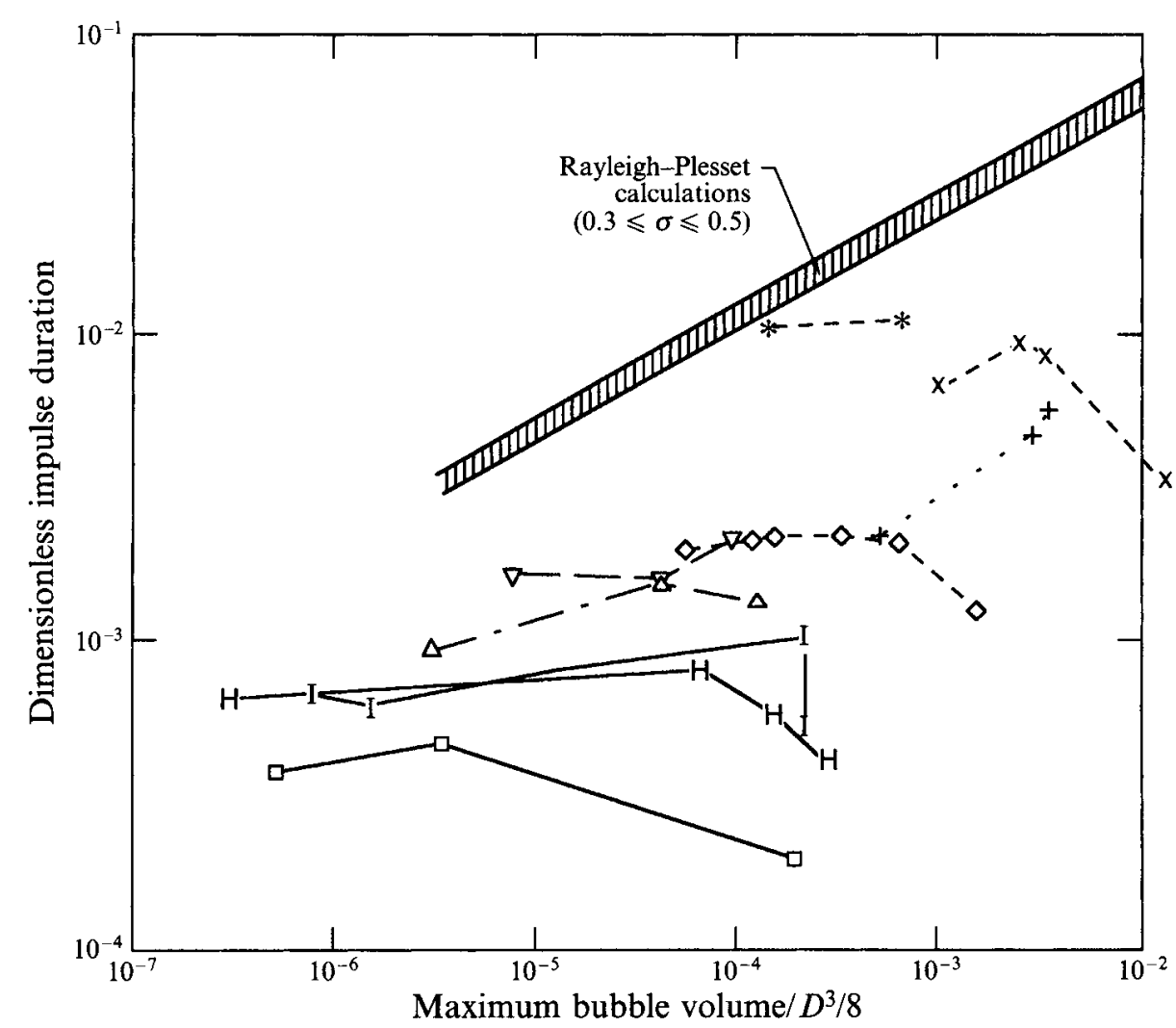

FIGURE 16. The dimensionless impulse duration, $\left(t_{2}-t_{1}\right) U / D$, as a function of maximum volume of the bubble (divided by $D^{3} / 8$ ). All the calculations using the Rayleigh-Plesset equation for $0.3 \leqslant \sigma \leqslant 0.5$ lay within the hatched region. The present experimental results are coded as in figure 14 .

There is, however, another effect which is present in the data of figures 14 and 15 . Virtually all of the data for a specific headform size and tunnel velocity tend first to increase as the cavitation number is reduced below the inception value. However, in almost all cases, this trend reaches a maximum at a particular cavitation number (or bubble volume) and begins to decrease with further reduction in $\sigma$. There are two effects which may contribute to this phenomenon. First, the noise or impulse may decrease due to the interactions between events as they become more numerous with decreasing $\sigma$. Second, the impulse may decrease due to a change in the dominant type of event as the $\sigma$ is decreased. When figures 11 and 14 are considered together, it is apparent that the impulses are maximum when the cavities cover about $20 \%$ of the surface area of the headform. Moreover, Arakeri \& Shanmuganathan (1985) reported that area void fractions larger than about $25 \%$ resulted in significant interactions between the bubbles and a reduction in the acoustic noise. In figure 14, the locations of the maxima appear to be shifted towards higher cavitation numbers at the lower velocities. This trend is consistent with that in figure 11 showing an increase in the area void fraction with decreasing velocity at the same cavitation numbers. The second effect, namely the change in the type of event, is discussed in the next section.

Average values of the dimensionless impulse duration, $\left(t_{2}-t_{1}\right) U / D$, are shown in figure 16 as a function of maximum volume of the bubble. The Rayleigh-Plesset calculations showed that these quantities are closely correlated for the relevant range 
of cavitation numbers and these results are also shown in figure 16. The results are similar to those for the acoustic impulse in the sense that they are about an order of magnitude smaller than the Rayleigh-Plesset results and exhibit somewhat similar variations with cavitation number. Most of the durations lay within the range of 15-40 $\mu \mathrm{s}$. Note that the response time of the hydrophone was of the order of $3 \mu \mathrm{s}$ and could therefore have affected these measurements.

\section{Effect of tails on bubble noise}

Comparisons of the hydrophone and electrode recordings for individual events led to several interesting conclusions. As stated earlier, when a bubble is located over a particular electrode (denoted by the index $i$ ) it modifies the electrical impedance in that vicinity; the instrumentation detects this change and converts it to a voltage signal $v_{i}(t)$ for that electrode. Figure 17 presents an example of the signals for the $50.8 \mathrm{~cm}$ diameter headform from the first and second patch electrodes (located at axial distances of $5.08 \mathrm{~cm}$ and $7.62 \mathrm{~cm}$ from the headform stagnation point). The corresponding noise signal is plotted on the same figure, time shifted by $170 \mu$ s which corresponds to the time necessary for the signal to travel from the headform surface to the hydrophone. The signals from a bubble with no streaks (figure $5 b$ ) and from a bubble developing attached streaks (figure $5 d$ ) are contrasted in this figure.

Simultaneous inspection of the electrode and hydrophone signals leads to several conclusions regarding the dynamics of collapse and the production of noise. Bubbles with streaks produce much longer electrode signals since the streaks continue to cover the electrode during their lifetime. Moreover, the trace from the first electrode will vanish before that from the second electrode, indicating that the collapse mechanism always proceeds in a downstream direction. Whether the cavity disappears by collapsing on the headform itself or detaches and is convected away by the flow is unclear. The time interval between the ends of the two electrode signals is often comparable to that measured for the case of unsheared travelling bubbles. This might suggest that the leading edge of the patch detaches first and the cavity is convected away by the flow while it is collapsing.

A characteristic time during which a bubble covers an electrode can be defined as

$$
\tau_{i}=\int_{0}^{e n d} v_{i}(t) \mathrm{d} t /\left(v_{i}\right)_{\max },
$$

where end denotes end of signal, and can be non-dimensionalized to define an electrode signal duration parameter, $\gamma_{i}=\tau_{i} U / D$. A bubble with attached streaks or patches will yield substantially larger $\gamma_{i}$ values than single unattached bubbles and, therefore, $\gamma_{i}$ provides a convenient indicator of the type of event which has occurred. Bubbles without attached tails were observed to have $\gamma$ values less than about 0.01 whereas bubbles with tails would yield values greater than 0.02 .

As might be expected, the duration parameters for individual electrodes were strongly correlated (Kuhn de Chizelle 1993). A long (or short) duration at the first electrode leads to a long (or short) duration at the second electrode. We conclude that tails only appear early in the bubble evolution and, if they do not appear, the bubble will continue without tails for the rest of its lifetime. This conclusion was confirmed by studies of photographs and video recordings.

By cross-correlating the electrode signals from the two first electrodes the bubble travel time, $t_{T}$, could be determined. This was found to be $t_{T}=0.043 \mathrm{D} / U$ regardless of the shape of the bubble. Thus the tails do not seem to significantly decrease the 

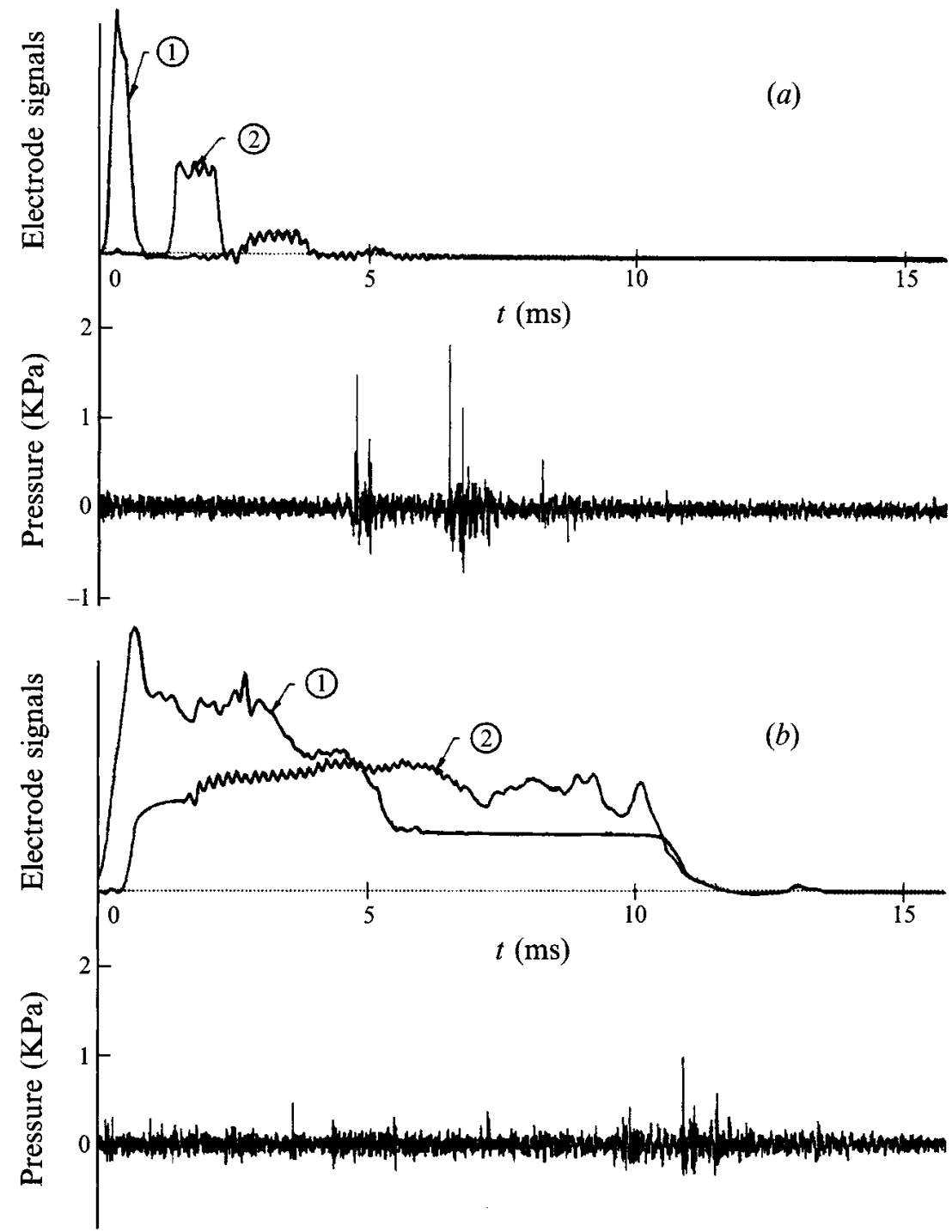

FIGURE 17. Typical electrode signals from the patch electrodes 1 and 2 and the corresponding hydrophone signals are shown for $(a)$ a bubble without tails and $(b)$ a bubble with tails.

bubble velocity. Furthermore potential flow calculations yielded the same fluid travel time, indicating that the bubble velocity is indistinguishable from that of the flow outside of the boundary layer.

Because of the strong correlation between $\gamma_{1}$ and $\gamma_{2}$, it is marginally better to use the geometric mean, $\gamma=\left(\gamma_{1} \gamma_{2}\right)^{1 / 2}$, as an indicator of the type of event. Since the electrodes and the hydrophone signals were recorded simultaneously, it is possible to correlate the acoustic output of each event with the $\gamma$ value for that event in order to explore the effect of tails on the noise. Figure 18 presents the acoustic impulse, $I$, as a function of the parameter, $\gamma$, for the $50.8 \mathrm{~cm}$ headform at $30 \%$ dissolved oxygen content. Most of the data are confined to cavitation numbers close to inception (low event rates) in order to ensure no overlap between events. Figure 18 leads to several conclusions. First we focus on the data on the left-hand side which has values of $\gamma$ less than 0.01 . This corresponds to unattached bubbles and for decreasing cavitation number both the 


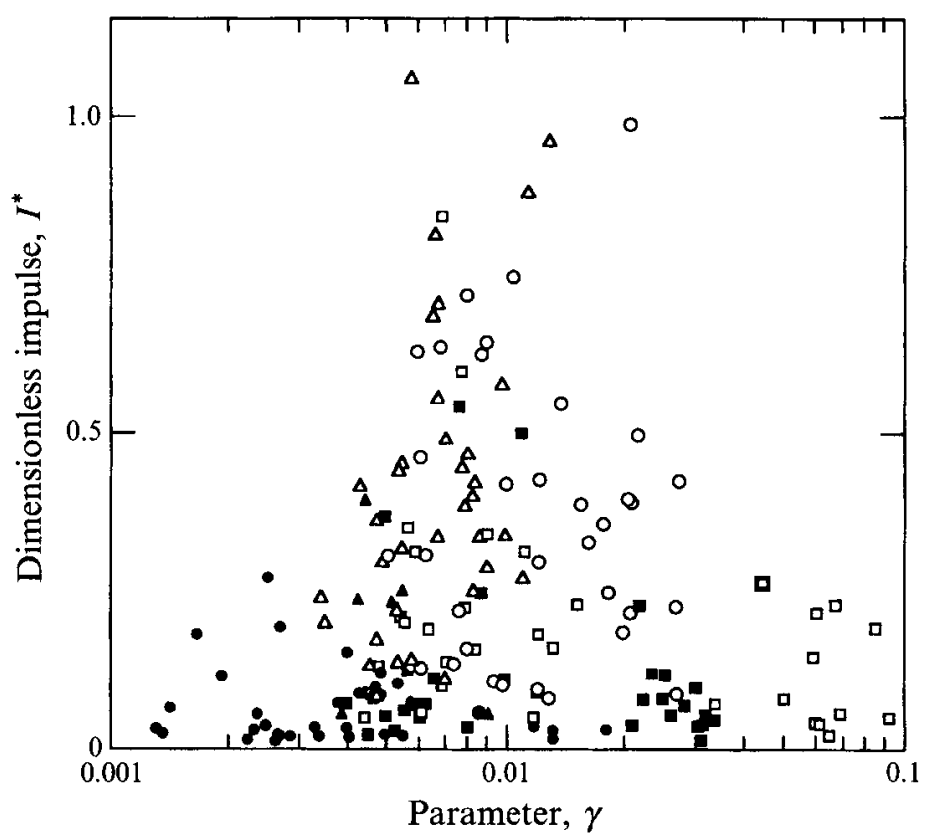

FIGURE 18. The acoustic impulse, $I$, as a function of the parameter, $\gamma$. Data shown are for the $50.8 \mathrm{~cm}$ headform diameter, $30 \%$ air content and at the following velocities and cavitation numbers: $U=15 \mathrm{~m} \mathrm{~s}^{-1}$ and $\sigma=0.64(\square), U=15 \mathrm{~m} \mathrm{~s}^{-1}$ and $\sigma=0.60(\square), U=11.5 \mathrm{~m} \mathrm{~s}^{-1}$ and $\sigma=0.71(0)$, $U=11.5 \mathrm{~m} \mathrm{~s}^{-1}$ and $\sigma=0.64(\bigcirc), U=9 \mathrm{~m} \mathrm{~s}^{-1}$ and $\sigma=0.78(\triangle)$, and $U=9 \mathrm{~m} \mathrm{~s}^{-1}$ and $\sigma=0.66(\triangle)$.

maximum impulse and the $\gamma$ value increase, leading to the rising envelope on the left of the figure. But the figure also shows a clear decline in the maximum impulse when the value of $\gamma$ exceeds about 0.02 . These $\gamma$ values correspond to bubbles which have tails or to attached patches and the figure shows that this results in a decrease in the impulse associated with the collapse of this type of event. The largest values of $\gamma$ correspond to the lowest cavitation numbers and thus to the largest patch cavities. The reduction in cavitation noise for attached bubbles and patches is probably due to the much less coherent and focused nature of the collapse of these structures.

\section{Conclusions}

In this paper we have presented results from a series of experiments carried out in the Large Cavitation Channel (LCC) of the David Taylor Research Center. The experiments were designed to investigate the scaling of the dynamics and acoustics of individual cavitation bubbles in flows around headforms. Many of the phenomena observed by Ceccio \& Brennen (1991) in experiments on $5.08 \mathrm{~cm}$ headforms were seen again in the present experiments. Such micro-fluid-mechanical phenomena included the spherical-cap shape of individual cavitation bubbles, the thin films separating the bubbles from the surface, the destabilization of that film, the occasional production of attached streaks in the wake of the bubbles and the complex process of bubble collapse involving bubble fission and roll-up.

Inception was observed to occur at substantially lower cavitation numbers on the larger headforms. One illustration of the difference was that, for the same air content and velocity, inception was observed to occur on the smallest headform at a cavitation number for which the cavitation on the largest headform was fully developed. Some of the differences in the appearance of individual bubbles on the three headforms could 
be attributed to this large difference in inception numbers since it implied quite different locations for the critical $C_{p}=-\sigma$ isobar. The most noticeable effect of scale on the appearance of cavitation was the increase in bubble-generated attached streaks and patches for the larger headforms. On the $5.08 \mathrm{~cm}$ headform a travelling bubble would occasionally generate two attached streaks or tails at the lateral extremes of the bubble. These would disappear almost immediately after the bubble collapsed. On the larger headforms at higher speeds (larger Reynolds numbers) and low cavitation numbers the streaks began to occur more frequently and extend behind the entire width of the bubble. The streaks would tend to produce a transient patch of attached cavitation which would disappear shortly after the bubble collapsed. For sufficiently small cavitation numbers, the patches would persist almost indefinitely and create larger attached cavitation structures. It is possible that this is the mechanism of formation for most patch and attached cavitation. Also the simultaneous coexistence and interaction of all the forms of cavitation structures over the large headforms is remarkable.

Another new observation during the present experiments was the appearance of a remarkable 'dimple' on the exterior surface of the travelling bubbles on the two larger headforms. These seem to be more pronounced when the bubble (or headform) is sufficiently large, which suggests that the surface tension tends to damp out the potential dimples on the smaller bubbles.

The experimental observations of the bubble shape, size and collapse location were also compared with the results of the 'travelling source' model of Kuhn de Chizelle \& Brennen (1993). This numerical method was developed to model the inviscid interactions between the bubble and the pressure gradients in the irrotational flow exterior to the boundary layer. Previous comparisons with the bubble profiles of Ceccio \& Brennen (1991) show that the exterior bubble shapes are qualitatively in agreement with those predicted by this approximate numerical procedure. In particular, the compression of the exterior surface during the growth phase and the evolution from a spherical-cap to a wedge shape are reproduced. Moreover, the calculations display a vestigal 'dimple' not dissimilar from whose observed experimentally. They did not, of course, reproduce the viscous phenomena such as the attached tails; more complex, viscous fluid modelling would be necessary for this purpose. However, a prerequisite for such a study is the availability of a solution for the irrotational flow outside the boundary layer.

Cavitation event rates were also evaluated from the photographs and videotapes and these data complement the observations of cavitation inception since inception was based on a chosen event rate. The event rates increase with increasing headform size and with decreasing cavitation number in the expected fashion if one assumes a fixed nuclei concentration. It is also demonstrated that the event rates appear to correspond to a nuclei population of the order of $0.1 \mathrm{~cm}^{-3}$ which is at least an order of magnitude lower than the expected nuclei population. We are continuing to investigate possible explanations for this discrepancy (Liu et al. 1993) including the bubble screening effect first suggested by Johnson \& Hsieh (1966).

The noise generated by individual events and the variations in the noise with the type of event were also investigated. The results demonstrate that the acoustic impulse generated by individual travelling bubbles scales with headform size and tunnel velocity and that this scaling is roughly in accord with that expected from the Rayleigh-Plesset or Fitzpatrick-Strasberg analysis. As expected, lower cavitation numbers lead to larger bubbles and larger impulses as long as the bubbles do not interfere with one another or with larger patch cavities. As in the previous study by 
Ceccio \& Brennen (1991) the impulses generated are substantially less than the magnitude predicted by the Rayleigh-Plesset analysis. It seems likely that the shearing and fission the bubble experiences prior to collapse lead to a less focused and therefore less 'efficient' noise-producing event. We have shown that the events which generate attached 'streaks' or 'tails' and which represent a greater fraction of the events at lower cavitation numbers and higher Reynolds numbers also produce significantly smaller acoustic impulses. This observation has clear implications for the scaling of cavitation noise.

At lower cavitation numbers, bubbles may trigger the formation of attached patches and the void fraction of bubbles in the cavitation region can reach a value at which bubble/bubble interactions become significant. Some of these interactions are quite complex. For example, when a travelling bubble encountered (or rode over) a patch cavity the dynamics of that bubble were altered and the acoustic output substantially diminished. It was also observed that a decrease in the noise appeared to occur when the area of the surface covered by cavitation bubbles and patches reached a value of about $20 \%$. This is in accord with the observation of Arakeri \& Shanmuganathan (1985). Both the bubble interaction effect and the change in the dominant type of event cause the decrease in the acoustic impulse at lower cavitation numbers.

Large-scale experiments like these require help of many people and the authors are very grateful to all of those who helped in this enterprise. We are very grateful to the ONR for their support under contracts N00014-91-J-1426 (S. L.C.) and N00014-91-J1295 (C.E.B., Y.K.deC.). We are also extremely grateful to the David Taylor Research Center (DTRC) and to their staff including W. B. Morgan for making the use of the LCC possible for us. From DTRC. Young Shen, Scott Gowing and James Blanton were important and valued members of the team who conducted the experiments. Po-Wen Yu (University of Michigan) was responsible for all the photographic aspects of the experiments, and Douglas Hart (Caltech) provided much assistance during the tests. The staff at the LCC in Memphis, Tennessee were remarkably tolerant and helpful and we wish to thank all of them most sincerely; we are particularly grateful to Bob Etter whose constant support was invaluable.

\section{REFERENCES}

ARakeri, V. H. \& Shanmuganathan, V. 1985 On the evidence for the effect of bubble interference on cavitation noise. J. Fluid Mech. 159, 131-150.

Benjamin, T. B. \& Ellis, A. T. 1966 The collapse of cavitation bubbles and the pressures thereby produced against solid boundaries. Phil. Trans. R. Soc. Lond. A 260, 221-240.

BILleT, M. L. \& Holl, J. W. 1979 Scale effects on various types of limited cavitation. ASME Intl Symp. on Cavitation Inception, pp. 11-24.

Blake, F. G. 1949 The onset of cavitation in liquids: I. Acoustics Res. Lab., Harvard Univ., Tech. Memo. 12.

BLAKE, W. K. 1986 Mechanics of Flow-induced Sound and Vibration. Academic.

Blake, W. K., Wolpert, M. J. \& Geib, F. E. 1977 Cavitation noise and inception as influenced by boundary-layer development on a hydrofoil. J. Fluid Mech. 80, 617-640.

Brennen, C. E. \& Ceccio, S. L. 1989 Recent observations on cavitation and cavitation noise. Proc. ASME 3rd Intl Symp. on Cavitation Noise and Erosion in Fluid Systems, FED 88, pp. 67-78.

Briançon-Marjollet, L., Franc, J. P. \& Michel, J. M. 1990 Transient bubbles interacting with an attached cavity and the boundary layer. J. Fluid Mech. 218, 355-376.

CECCIO, S. L. 1990 Observations of the dynamics and acoustics of travelling bubble cavitation. $\mathrm{PhD}$ thesis, California Institute of Technology. 
Ceccio, S. L. \& Brennen, C. E. 1991 Observations of the dynamics and acoustics of travelling bubble cavitation. $J$. Fluid Mech. 233, 633-660.

Chahine, G., Duraiswami, R. \& Rebut, M. 1992 Analytical and numerical study of large bubble/bubble and bubble/flow interactions. Proc 19th Symp. on Naval Hydrodynamics, Seoul, Korea, pp. 679-699.

ELLIS, A. T. 1952 Observations on cavitation bubble collapse. Calif. Inst. Tech. Hydr. Lab. Rep. 21-12.

FitzPatrick, H. M. \& Strasberg, M. 1956 Hydrodynamic sources of sound. Proc. First ONR Symp. on Naval Hydrodynamics, pp. 241-280. National Academy Press.

FuJiKaWA, S. \& AKamaTsu, T. 1980 Effects of the non-equilibrium condensation of vapour on the pressure wave produced by the collapse of a bubble in a liquid. J. Fluid Mech. 97, 481-512.

GILMORE, F. R. 1952 The collapse and growth of a spherical bubble in a viscous compressible liquid. Calif. Inst. Tech. Hydr. Lab. Rep. 26-4.

Hamilton, M. F., Thompson, D. E. \& Billet, M. L. 1982 An experimental study of traveling bubble cavitation and noise. Proc. ASME Intl Symp. on Cavitation Noise, pp. 25-33.

Hickling, R. \& Plesset, M. S. 1964 Collapse and rebound of a spherical bubble in water. Phys. Fluids 7, 7-14.

Holl, J. W. \& CARRoll, J. A. 1979 Observations of the various types of limited cavitation on axisymmetric bodies. Proc. ASME Intl Symp. on Cavitation Inception, pp. 87-99.

Holl, J. W. \& KorNHAUSER, A. L. 1970 Thermodynamic effects on desinent cavitation on hemispherical nosed bodies in water at temperatures from $80^{\circ} \mathrm{F}$ to $260^{\circ} \mathrm{F}$. Trans. $A S M E \mathrm{D}$ : J. Basic Engng 92, 44-58.

Holl, J. W. \& WisLICENUS, G. F. 1961 Scale effects on cavitation. Trans. ASME D: J. Basic Engng 83, 385-398.

Huang, T. T. 1979 Cavitation inception observations on six axisymmetric headforms. Proc. ASME Intl Symp. on Cavitation Inception, pp. 51-61.

Johnson, V. E. \& HsIEH, T. 1966 The influence of the trajectories of gas nuclei on cavitation inception. Proc. 6th ONR Symp. on Naval Hydrodynamics, pp. 163-182.

KellogG, O. D. 1953 Foundations of Potential Theory. Dover.

Kiмото, H. 1987 An experimental evaluation of the effects of a water microjet and a shock wave by a local pressure sensor. Intl ASME Symp. on Cavitation Res. Facilities and Techniques, FED 57, pp. 217-224.

KNAPP, R. T. \& HollandeR, A. 1948 Laboratory investigations of the mechanisms of cavitation. Trans. ASME 70, 419.

KueTHE, A. M. \& Chow, C. Y. 1986 Foundations of Aerodynamics. John Wiley and Sons.

KuHN DE CHIzelle, Y. 1993 Hydrodynamics, acoustics and scaling of traveling bubble cavitation. $\mathrm{PhD}$ thesis, California Institute of Technology.

KuHN DE Chizelle, Y. \& Brennen, C. E. 1993 Comparison of observed and calculated shapes of travelling cavitation bubbles. Proc. IUTAM Symp. on Bubble Dynamics and Interface Phenomena, Birmingham, UK, pp. 207-217.

Kuhn de Chizelle, Y., Ceccio, S. L., Brennen, C. E. \& Gowing, S. 1992 a Scaling experiments on the dynamics and acoustics of travelling bubble cavitation. Proc. 3rd I. Mech. E. Intl Conf. on Cavitation, Cambridge, UK, pp. 165-170.

Kuhn de Chizelle, Y., Ceccio, S. L., Brennen, C. E. \& Shen, Y. $1992 b$ Cavitation scaling experiments with headforms: bubble acoustics. Proc. 19th Symp. on Naval Hydrodynamics, Seoul, Korea, pp. 72-84. National Academy Press.

Kumar, S. \& Brennen, C. E. 1993 A study of pressure pulses generated by travelling bubble cavitation. J. Fluid Mech. 255, 541-564.

LAUTERBORN, W. \& BOLLE, H. 1975 Experimental investigations of cavitation bubble collapse in the neighbourhood of a solid boundary. J. Fluid Mech. 72, 391-399.

LindGren, H. \& JoHnsson, C. A. 1966 Cavitation inception on headforms. ITTC comparative experiments. Proc. 11th Intl Towing Tank Conf., Tokyo, pp. 219-232.

Liu, Z., Kunn de Chizelle, Y. \& Brennen, C. E. 1993 Cavitation event rates and nuclei distributions. Proc. ASME Symp. on Cavitation Inception, FED 177, pp. 13-24.

MeUlen, J. H. J. VAN DER \& ReNESSE, R. L. VAN 1989 The collapse of bubbles in a flow near a 
boundary. Proc. 17th Symp. on Naval Hydrodynamics, The Hague, pp. 195-217. National Academy Press.

MeYer, R. S., BiLLET, M. L. \& Holl, J. W. 1989 Freestream nuclei and traveling-bubble cavitation. Trans. ASME I: J. Fluids Engng 114, 672-679.

Morgan, W. B. 1990 David Taylor Research Center's large cavitation channel. Proc. 19th Intl Towing Tank Conf., Madrid, Vol. 2, pp. 419-427.

NAUDE, C. F. \& Ellis, A. T. 1961 On the mechanism of cavitation damage by non-hemispherical cavities in contact with a solid boundary. Trans. ASME D: J. Basic Engng 83, 648-656.

O'Hern, T., D'Agostino, L. \& Acosta, A. J. 1988 Comparison of holographic and counter measurements of cavitation nuclei in the ocean. Trans. ASME I: J. Fluids Engng 110, 200-207.

ParkIn, B. R. 1952 Scale effects in cavitating flow. PhD thesis, California Institute of Technology.

Plesset, M. S. 1949 The dynamics of cavitation bubbles. Trans. ASME E: J. Appl. Mech. 16, 228-231.

RAYLEIGH, LORD 1917 On the pressure developed in a liquid during the collapse of a spherical cavity. Phil. Mag. 34, 94-98.

SCHIEBE, F. R. 1972 Measurements of the cavitation susceptibility of water using standard bodies. St. Anthony Falls Hydr. Lab., U. of Minnesota, Rep. 118.

Shen, Y. T., GowIng, S. \& PierCe, R. D. 1984 Cavitation susceptibility measurements by a venturi. Proc. ASME Intl Symp. on Cavitation Inception, pp. 9-18.

Shima, A., Takayama, K., Tomita, Y. \& Miura, N. 1981 An experimental study of effects of a solid wall on the motion of bubbles and shock waves in bubble collapse. Acustica 48, 293-301.

Shima, A., Takayama, K., Tomita, Y. \& OhSAwa, N. 1983 Mechanism of impact pressure generation from spark-generated bubble collapse near a wall. $A I A A J .21,55-69$.

Vogel, A., LaUterborn, W. \& Timm, R. 1989 Optical and acoustic investigations of dynamics of the laser-produced cavitation bubbles near a solid boundary layer. J. Fluid Mech. 206, 299-338. 